\title{
Pencils on real curves
}

\author{
Marc Coppens $^{* 1}$ and Johannes Huisman**2 \\ ${ }^{1}$ Katholieke Hogeschool Kempen, Departement Industrieel Ingenieur en Biotechniek, KU Leuven, Dept. \\ Wiskunde Groep Algebra, Kleinhoefstraat 4, B 2440 Geel, Belgium \\ ${ }^{2}$ Université Européenne de Bretagne, France, Université de Brest, CNRS, UMR 6205, Laboratoire de \\ Mathématiques de Brest, ISSTB, 6, avenue Victor Le Gorgeu, CS 93837, 29238 Brest Cedex 3, France
}

Received 23 August 2011, revised 14 March 2012, accepted 10 September 2012

Published online 11 January 2013

Key words Real curve, linear pencil, topological degree, morphism, Brill-Noether Theory, divisor MSC (2010) 14H51, 14P99

We consider coverings of real algebraic curves to real rational algebraic curves. We show the existence of such coverings having prescribed topological degree on the real locus. From those existence results we prove some results on Brill-Noether Theory for pencils on real curves. For coverings having topological degree $\underline{0}$ we introduce the covering number $k$ and we prove the existence of coverings of degree 4 with prescribed covering number.

(c) 2013 WILEY-VCH Verlag GmbH \& Co. KGaA, Weinheim

\section{Introduction}

In this paper we show the existence of coverings $\pi: X \rightarrow \mathbb{P}^{1}$ of $\mathbb{P}^{1}$ by real curves satisfying some specific properties related to the real locus $X(\mathbb{R})$. Part of those constructions is related to recent results on real pencils (one-dimensional linear systems) on real curves.

We write $X(\mathbb{C})$ to denote the associated Riemann surface, $g(X)$ to denote its genus and $a(X) \in\{0,1\}$ such that $2-a(X)$ is the number of connected components of $X(\mathbb{C}) \backslash X(\mathbb{R})$. Consider a covering $\pi: X \rightarrow \mathbb{P}^{1}$ and choose an orientation on $\mathbb{P}^{1}(\mathbb{R})$. Let $C$ be a connected component of $X(\mathbb{R})$ and consider the restriction $\left.\pi\right|_{C}: C \rightarrow \mathbb{P}^{1}(\mathbb{R})$. Fixing an orientation on $C$ this restriction has a topological degree $\delta_{C}(\pi)$ and we assume the orientations are chosen such that $\delta_{C}(\pi) \geq 0$. We consider the existence of coverings having prescribed values for those topological degrees. There are trivial natural restrictions between $d=\operatorname{deg}(\pi), g=g(X), a=a(X)$ and those topological degrees and we prove that there exists such a covering $\pi$ for all values $d, g, a$ and $\delta_{C}$ satisfying those restrictions.

In particular, for each $s \geq 2, g \equiv s-1(\bmod 2)$ with $g \geq s-1$ there exists a real curve $X$ of genus $g$ such that $a(X)=0$ and $X(\mathbb{R})$ has exactly $s$ connected components and a morphism $\pi: X \rightarrow \mathbb{P}^{1}$ of degree $s$ such that $\delta_{C}(\pi)=1$ for each component $C$ of $X(\mathbb{R})$. In particular $\pi(\mathbb{R}): X(\mathbb{R}) \rightarrow \mathbb{P}^{1}(\mathbb{R})$ is the union of $s$ homeomorphisms. In [21] one introduces very special linear systems on real curves satisfying strong Cliffordtype properties for real curves and in Proposition 2.1 of [21] it is proved that the only very special pencils are exactly those defined by such coverings. So we obtain a proof for the existence of very special pencils of all possible types.

In [9] one studies some Brill-Noether properties for pencils on real curves. We give another proof of part of the results in [9] making intensive use of results coming from the theory of complex curves. In this way it is enough to prove the existence of one real curve having a pencil of suited degree. Because of our existence results we obtain Brill-Noether properties for pencils having prescribed topological degree on the connected components of the real locus. While the arguments in [9] are restricted to real curves having real points, our arguments also give Brill-Noether properties for pencils on real curves without real points.

\footnotetext{
* Corresponding author: e-mail: marc.coppens@khk.be, Phone: +32 16327011, Fax: +32 16327998

** e-mail: johannes.huisman@ univ-brest.fr, Phone: +33(0)298016194, Fax: +33(0)298016790
} 
The existence and Brill-Noether results are also considered in [5], [6] and [7] without considering the topological degrees. In order to make the arguments more careful with respect to dimension arguments on the real locus of a variety defined over $\mathbb{R}$ we use universal spaces representing morphisms that are known to be globally smooth because of Horikawa deformation theory.

A real divisor $D$ on a real curve is called totally non-real in case the support of $D$ contains no real point of $X$. It is known that for a real linear system on a real curve the parity of the restriction of a divisor to a connected component of $X(\mathbb{R})$ is constant. Hence if this parity is odd for some component then the linear system does not contain a totally non-real divisor. Restricting to linear systems such that the parity is even for all components of $X(\mathbb{R})$, there is a sharp result in [11] concerning the existence of totally non-real divisors in such a linear system. For linear systems of large dimension, it is not easy to find non-trivial examples of such linear systems having no totally non-real divisors. In the case of pencils our constructions give a lot of such examples coming from coverings having only even topological degrees and at least one of them being non-zero. One can ask for the existence of such examples coming from coverings with all topological degrees equal to zero. We prove the existence of all types of such coverings of the smallest possible degree 4.

In Section 1 we give the constructions that are the base for the existence of coverings with prescribed topological degrees. In Section 2 we prove the existence of coverings with prescribed topological degrees. In Section 3 we discuss Brill-Noether problems for pencils on real curves. Finally in Section 4 we prove the existence of coverings of degee 4 with all topological degrees equal to 0 and having no totally non-real divisor.

Terminology 1.1 A smooth real curve $X$ of genus $g=g(X)$ is a scheme defined over $\mathbb{R}$ such that the base change $X \times_{\mathbb{R}} \mathbb{C}$, denoted by $X_{\mathbb{C}}$, is a complete connected smooth complex curve of genus $g$. We also write $X(\mathbb{C})$ to denote the set of closed points of $X_{\mathbb{C}}$ and we consider it as a Riemann surface of genus $g$. As usual the nontrivial element of $\operatorname{Gal}(\mathbb{C} / \mathbb{R})$ is denoted by $z \rightarrow \bar{z}$ and it is called (complex) conjugation. For a real curve $X$ it induces an $\mathbb{R}$-involution $\sigma: X_{\mathbb{C}} \rightarrow X_{\mathbb{C}}$. For $P \in X(\mathbb{C})$ we write $\bar{P}$ instead of $\sigma(P)$ and we call it the conjugated point of $P$. In case $P=\bar{P}$ then $P$ is called a real point of $X$. In case $P \neq \bar{P}$ then we call $P+\bar{P}$ a non-real point of $X$. The set of real points is called the real locus $X(\mathbb{R}) \subset X(\mathbb{C})$. This locus is a union of $s(X)$ connected components, each one of them homeomorphic to a circle. The invariant $a(X)$ is defined by $a(X)=1$ in case $X(\mathbb{C}) \backslash X(\mathbb{R})$ is connected and otherwise $a(X)=0$ (in that case $X(\mathbb{C}) \backslash X(\mathbb{R})$ has two connected components). We say a smooth real curve $X$ has topological type $(g, s, a)$ if $g(X)=g, s(X)=s$ and $a(X)=a$. By a theorem of Weichold such real curve exists if and only if either $a=1$ and $0 \leq s \leq g$ else $a=0, s \equiv g+1(\bmod 2)$ and $1 \leq s \leq g+1$ (see [25], see also [19, Theorem 5.3]). A triple $(g, s, a)$ satisfying those restrictions is called an admissible topological type for real curves.

For a divisor $D=\sum_{i=1}^{n} m_{i} P_{i}$ on $X_{\mathbb{C}}$ we write $\bar{D}=\sum_{i=1}^{n} m_{i} \overline{P_{i}}$ and we call it the conjugated divisor of $D$. As usual $\sum_{i=1}^{n} m_{i}$ is called the degree of $D$, denoted by $\operatorname{deg}(D)$. We say $D$ is a real divisor if $\bar{D}=D$. If we say $D$ is a divisor on $X$ then we mean it is a real divisor on $X_{\mathbb{C}}$. We write $\sim$ to denote linearly equivalence of divisors.

Let $L$ be an invertible sheaf on $X_{\mathbb{C}}$. It can be described by means of trivializations on an open covering $\left(U_{i}\right)_{i \in I}$ of $X(\mathbb{C})$ and transition functions $a_{i, j}$ (those are regular functions $U_{i} \cap U_{j} \rightarrow \mathbb{C}^{*}$ ). The conjugated invertible sheaf $\bar{L}$ is defined by means of trivializations on the open covering $\left(\sigma\left(U_{i}\right)\right)_{i \in I}$ and transition functions $\overline{a_{i, j}}$ (for $P \in \sigma\left(U_{i} \cap U_{j}\right)$ one has $\left.\overline{a_{i, j}}(P)=\overline{a_{i, j}(\bar{P})}\right)$. Each invertible sheaf $L$ on $X_{\mathbb{C}}$ is isomorphic to $\mathcal{O}_{X_{\mathbb{C}}}(D)$ for some divisor $D$ on $X_{\mathbb{C}}$. In case $k=\operatorname{deg}(D)$ then we say $L$ is an invertible sheaf of degree $k$ and we write $\operatorname{deg}(L)=k$. In case $L \cong \mathcal{O}_{X}(D)$ then $\bar{L} \cong \mathcal{O}_{X}(\bar{D})$.

We say $L$ is a real invertible sheaf if $L \cong \mathcal{O}_{X}(D)$ for some real divisor $D$. We say $L$ is invariant under conjugation if $L \cong \bar{L}$. In particular a real invertible sheaf is invariant under conjugation. Let $\operatorname{Pic}(X)$ be the Picard scheme of $X$ defined over $\mathbb{R}$ (see [15]). Then $\operatorname{Pic}(X)_{\mathbb{C}}$ represents the Picard functor on $X_{\mathbb{C}}$, in particular $\operatorname{Pic}(X)(\mathbb{C})$ parameterizes invertible sheaves on $X_{\mathbb{C}}$. For $k \in \mathbb{Z}$ one has natural subschemes $\operatorname{Pic}^{k}(X)$ such that $\operatorname{Pic}^{k}(X)(\mathbb{C})$ parameterizes invertible sheaves of degree $k$ on $X_{\mathbb{C}}$. The real locus $\operatorname{Pic}^{k}(X)(\mathbb{R})$ parameterizes invertible sheaves of degree $k$ on $X_{\mathbb{C}}$ invariant under conjugation. Let $\mathrm{Pic}^{k}(X)(\mathbb{R})^{+}$be the sublocus parameterizing real invertible sheaves. In case $X(\mathbb{R}) \neq \emptyset$ then $\operatorname{Pic}(X)$ also represents the Picard functor on $X$ and therefore $\operatorname{Pic}^{k}(X)(\mathbb{R})^{+}=\operatorname{Pic}^{k}(X)(\mathbb{R})$ in that case. In case $X(\mathbb{R})=\emptyset$ then $\operatorname{Pic}^{k}(X)(\mathbb{R})^{+}$is a subgroup of $\operatorname{Pic}^{k}(X)(\mathbb{R})$ with quotient $\mathbb{Z} / 2 \mathbb{Z}$. For $L \in \operatorname{Pic}^{k}(X)(\mathbb{R})$ with $L \notin \operatorname{Pic}(X)(\mathbb{R})^{+}$one has $\operatorname{deg}(L) \equiv g-1(\bmod 2)($ see $[14$, Proposition 2.2]). 
We write $|L|$ to denote the complete linear system of an invertible sheaf $L$ on $X_{\mathbb{C}}$. In case $L$ is a real invertible sheaf then $|L|(\mathbb{R})$ is the space of real divisors contained in $|L|$. One has $|L|=|L|(\mathbb{R}) \otimes \mathbb{C}$ in a natural way (meaning $|L|(\mathbb{R})$ can be considered as a projective space $\mathbb{P}^{r}$ and then $|L|=\mathbb{P}_{\mathbb{C}}^{r}$ ). As usual we write $g_{d}^{r}$ to denote a linear system of dimension $r$ and degree $k$ on $X_{\mathbb{C}}$. In case $L$ is a real invertible sheaf of degree $k$ then we say a linear subsystem $g_{k}^{r}$ of $|L|$ is defined over $\mathbb{R}$ if there exists a $k$-dimensional linear subsystem $g_{k}^{r}(\mathbb{R}) \subset|L|(\mathbb{R})$ such that $g_{k}^{r}=g_{k}^{r}(\mathbb{R}) \otimes \mathbb{C}$. In such case we say $g_{k}^{r}$ is a linear system on $X$. In particular if $L$ is a real invertible sheaf then we say $|L|$ is a linear system on $X$.

We write $\mathbb{P}^{1}$ to denote the projective line defined over $\mathbb{R}$ and we write $R_{0}$ to denote the smooth real curve of genus 0 without real points (defined by the equation $X^{2}+Y^{2}+Z^{2}=0$ in $\mathbb{P}^{2}$ ).

Let $X$ be a smooth real curve. A covering $\pi: X \rightarrow \mathbb{P}^{1}$ or $\pi: X \rightarrow R_{0}$ is a finite morphism defined over $\mathbb{R}$. We say $\pi$ has degree $d$ in case the associated covering $\pi_{\mathbb{C}}: X_{\mathbb{C}} \rightarrow \mathbb{P}_{\mathbb{C}}^{1}$ has degree $d$. Such covering corresponds to a base point free pencil $g_{d}^{1}$ on $X_{\mathbb{C}}$. In case of $\pi: X \rightarrow \mathbb{P}^{1}$ this is called a real pencil on $X$. In case of $\pi: X \rightarrow R_{0}$ this is called a non-real invariant pencil on $X$. This case only can occur if $X(\mathbb{R})=\emptyset$ (X has no real points) and moreover, in this case the pencil has no real divisor but it is invariant under complex conjugation. We write $\pi(\mathbb{C}): X(\mathbb{C}) \rightarrow \mathbb{P}^{1}(\mathbb{C})$ to denote the covering of Riemann surfaces. In case $X(\mathbb{R})$ is not empty we write $\pi(\mathbb{R}): X(\mathbb{R}) \rightarrow \mathbb{P}^{1}(\mathbb{R})$ to denote the restriction of $\pi(\mathbb{C})$ to the real locus $X(\mathbb{R})$. For a connected component $C$ of $X(\mathbb{R})$ we write $\pi_{C}: C \rightarrow \mathbb{P}^{1}(\mathbb{R})$ to denote the restriction of $\pi(\mathbb{R})$ to $C$. On $\mathbb{P}^{1}(\mathbb{R})$, homeomorphic to a circle $S^{1}$, we choose an orientation and then for $C$, also homeomorphic to $S^{1}$, we choose the orientation such that the degree of $\pi_{C}$, denoted by $\delta_{C}(\pi)$, is nonnegative. If $P \in C$ and $\pi_{C}$ is not ramified at $P$, then the local degree $\delta_{P}(\pi)$ is equal to 1 (resp. -1 ) if $\pi_{C}$ preserves (resp. reverses) the orientation locally at $P$. Let $C_{1}, \ldots, C_{s}$ be the connected components of $X(\mathbb{R})$ and let $\delta_{i}=\delta_{C_{i}}(\pi)$ for $1 \leq i \leq s$. We can always assume $\delta_{1} \geq \delta_{2} \geq \cdots \geq \delta_{s}$ and then we say $\pi$ has topological degree $\left(\delta_{1}, \delta_{2}, \ldots, \delta_{s}\right)$ (shortly denoted by $\left.\underline{\delta}\right)$.

\section{Constructions}

The proof of the existence of coverings with prescribed topological degrees uses an induction argument. To make that argument we start with a smooth real curve $Y$ and a suited covering $\pi_{Y}: Y \rightarrow \mathbb{P}^{1}$. Using this covering we construct a covering $\pi_{0}: X_{0} \rightarrow \mathbb{P}^{1}$ with $X_{0}$ being a real singular nodal curve and we use a real smoothing $\pi_{t}: X_{t} \rightarrow \mathbb{P}^{1}$ of $\pi_{0}$. In this part we describe those constructions giving relations between topological degrees of $\pi_{Y}$ and of $\pi_{t}$. This will be the base for the induction argument in the next section.

In those constructions we start by taking local smoothings of the nodes of $X_{0}(\mathbb{C})$ having a natural antiholomorphic involution. Those local smoothings glue with the complement $V$ of a neighborhood of the nodes of $X_{0}(\mathbb{C})$ giving rise to a deformation of compact Riemann surfaces $X_{t}(\mathbb{C})$ and holomorphic coverings $\pi_{t}(\mathbb{C})$ : $X_{t}(\mathbb{C}) \rightarrow \mathbb{P}^{1}(\mathbb{C})$. This complement $V$ can be taken to be invariant under complex conjugation on $X_{0}(\mathbb{C})$ and this fits with the antiholomorphic involution of the local smoothings under the gluing. Hence we obtain an antiholomorphic involution $\sigma_{t}$ on $X_{t}(\mathbb{C})$. It is well-known that this defines a smooth real curve $X_{t}$ inducing the Riemann surface $X_{t}(\mathbb{C})$ such that $\sigma_{t}$ corresponds to complex conjugation (see e.g. [19, Section 4]). Moreover the morphism $\pi_{t}(\mathbb{C}): X_{t}(\mathbb{C}) \rightarrow \mathbb{P}^{1}(\mathbb{C})$ is invariant under complex conjugation hence it comes from a morphism $\pi_{t}: X_{t} \rightarrow \mathbb{P}^{1}$ (indeed, the graph of $\pi_{t}(\mathbb{C})$ is a closed subspace of $\left(X_{t} \times \mathbb{P}^{1}\right)(\mathbb{C})$ invariant under complex conjugation on $\left.X_{t} \times \mathbb{P}^{1}\right)$.

\subsection{Construction I}

Let $Y$ be a real curve of genus $g$ and let $\pi: Y \rightarrow \mathbb{P}^{1}$ be a morphism of degree $k$ defined over $\mathbb{R}$. Assume $C$ is a connected component of $Y(\mathbb{R})$ and let $P$ be a point of $C$ such that $\delta_{P}(\pi)=1$ (such point $P$ does exist because $\left.\delta_{C}(\pi) \geq 0\right)$. Take a copy of $\mathbb{P}^{1}$ and consider the singular curve $X_{0}=Y \cup_{P} \mathbb{P}^{1}$ obtained by identifying $P$ on $Y$ with $\pi(P)$ on $\mathbb{P}^{1}$. This singular curve has a natural morphism $\pi_{0}$ defined over $\mathbb{R}$ of degree $k+1$ to $\mathbb{P}^{1}$ having restriction $\pi$ to $Y$ and the identity to $\mathbb{P}^{1}$ (see Figure 1).

Locally at $P$ this situation can be described inside $\mathbb{C}^{2}$ such that the curve $X_{0}$ has equation $x^{2}-y^{2}=0$ and the morphism is locally defined by $(x, y) \rightarrow x$ and we can assume the coordinates are compatible with the real structure on $X_{0}$ (this means complex conjugation on $X_{0}$ corresponds to complex conjugation in $\mathbb{C}^{2}$ ). Let $U$ be a small neighborhood of $(0,0)$ in $\mathbb{C}^{2}$ and $V \subset U$ a much smaller one and let $U_{0}=U \cap X_{0}, V_{0}=V \cap X_{0}$. For $Q \neq P$ in $U_{0}$ we can use $x$ to define a holomorphic coordinate at $Q$. Consider a local deformation of $X_{0}$ 


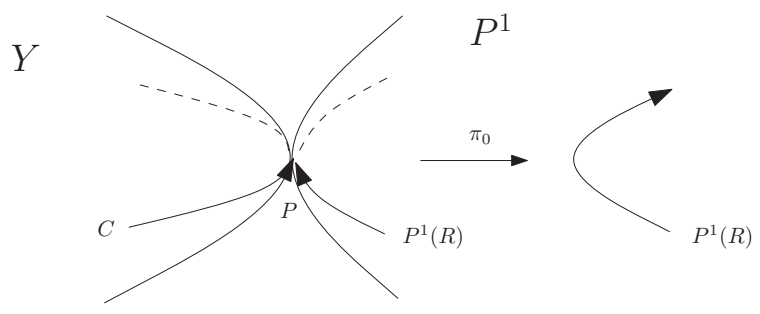

Fig. 1 Construction I, the curve $X_{0}$.

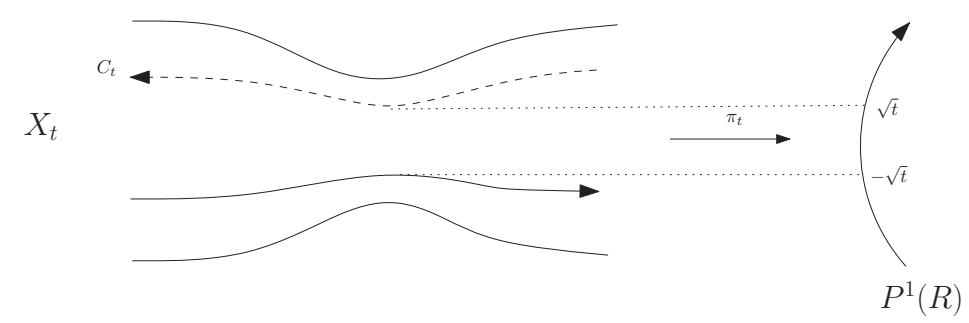

Fig. 2 Construction I, deformation with real ramification.

at $P$ defined by $x^{2}-y^{2}=t$ with $t \in \mathbb{R}\left(|t|\right.$ very small) and let $U_{t}$ (resp. $V_{t}$ ) be the intersection with $U$ (resp. $V)$. We use a gluing of $U_{t}$ and $X_{0}(\mathbb{C}) \backslash V_{0}$ as follows. For $z_{0} \in \mathbb{C}^{*}$ let $z_{0} \sqrt{z}$ be the locally defined holomorphic square root function such that $z_{0} \sqrt{z_{0}^{2}}=z_{0}$. A point $Q \in U_{0} \backslash V_{0}$ has coordinates $(x, x)$ or $(x,-x)$. We identify $(x, x)$ with $\left(x, x_{x} \sqrt{x^{2}-t}\right)$ and $(x,-x)$ with $\left(x,-{ }_{x} \sqrt{x^{2}-t}\right)$. (One should adapt the description of $V_{t}$ and $U_{t}$ to this identification.) This defines a Riemann surface $X_{t}(\mathbb{C})$. On $U_{t}$ we define $\sigma_{t}(x, y)=(\bar{x}, \bar{y})$. We need to show this behaves well under the previous identification. Since ${ }_{x} \sqrt{x^{2}-t}$ is close to $x$ one has $\bar{x} \sqrt{x^{2}-t}$ is close $\bar{x}$. Moreover

$$
\left.\overline{\left({ }_{x} \sqrt{x^{2}-t}\right.}\right)^{2}=\overline{\left({ }_{x} \sqrt{x^{2}-t}\right)^{2}}=\overline{x^{2}-t}=\bar{x}^{2}-t
$$

hence

$$
\overline{{ }_{x} \sqrt{x^{2}-t}}=\bar{x} \sqrt{\bar{x}^{2}-t} .
$$

Hence under the identification $(\bar{x}, \bar{x})$ is identified with $\left(\bar{x}, \overline{{ }_{x} \sqrt{x^{2}-t}}\right)$ and similarly $(\bar{x},-\bar{x})$ is identified with $\left(\bar{x}, \overline{-{ }_{x} \sqrt{x^{2}-t}}\right)$. As mentioned at the beginning of this section we obtain a real smooth curve $X_{t}$ and a covering $\pi_{t}: X_{t} \rightarrow \mathbb{P}^{1}$ of degree $k+1$. Under this deformation the union $C \cup_{P} \mathbb{P}^{1}(\mathbb{R})$ deforms to a connected component $C_{t}$ of $X_{t}(\mathbb{R})$

In case $t>0$ the morphism $\pi_{t}$ has ramification on $C_{t}$ above $x= \pm \sqrt{t}$. We call this the deformation with real ramification. In order to have an orientation on $C_{t}$ we have to change the orientation on the attached $\mathbb{P}^{1}(\mathbb{R})$. This implies that $\delta_{C_{t}}\left(\pi_{t}\right)=\delta_{C}(\pi)-1$. Of course, if $\delta_{C}(\pi)=0$ we also change the orientation of $C_{t}$ in order to obtain $\delta_{C_{t}}\left(\pi_{t}\right)=1$ (see Figure 2).

In case $t<0$ the morphism $\pi_{t}$ has no ramification on $C_{t}$ close to $P$. We call this the deformation without real ramification. We use the orientation on $C_{t}$ obtained from both the orientation on $C$ and the attached $\mathbb{P}^{1}(\mathbb{R})$. This implies $\delta_{C_{t}}\left(\pi_{t}\right)=\delta_{C}(\pi)+1$ (see Figure 3).

In both cases $X_{t}(\mathbb{C}) \backslash X_{t}(\mathbb{R})$ is connected if and only if $X(\mathbb{C}) \backslash X(\mathbb{R})$ is connected.

\subsection{Construction II}

Let $Y$ be a real curve of genus $g$ and let $\pi: Y \rightarrow \mathbb{P}^{1}$ be a morphism of degree $k$ defined over $\mathbb{R}$. Let $Q \in \mathbb{P}^{1}(\mathbb{R})$ not a branch point of $\pi$ and assume $P+\bar{P} \subset \pi^{-1}(Q)$, a non-real point on $Y$ (in particular we assume such non-real point exists). Let $X_{0}$ be the singular curve obtained from $Y$ by identifying $P$ with $\bar{P}$. This singular curve has a natural morphism of degree $k$ to $\mathbb{P}^{1}$ defined over $\mathbb{R}$ obtained from $\pi$ (see Figure 4). 


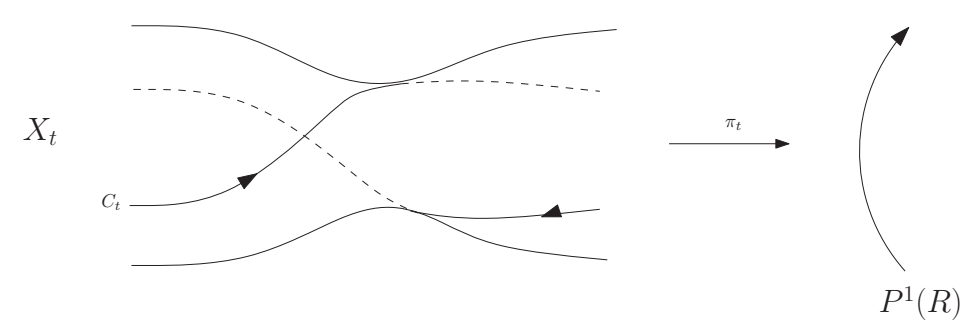

Fig. 3 Construction I, deformation without real ramification.

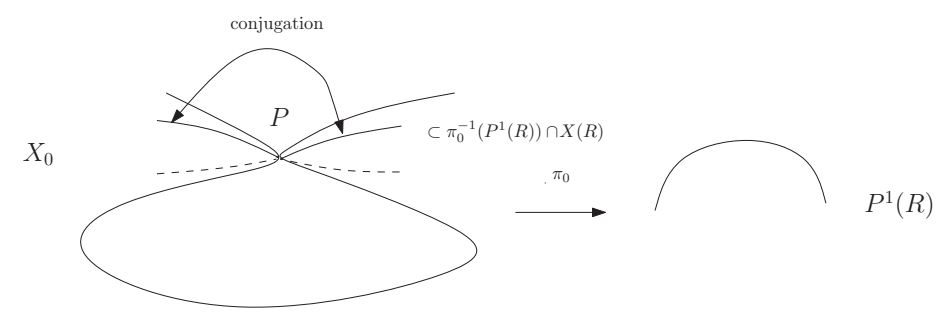

Fig. 4 Construction II, the curve $X_{0}$.

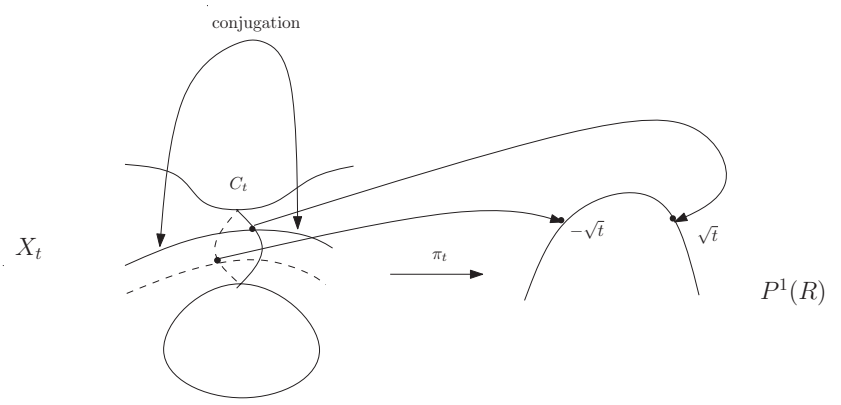

Fig. 5 Construction II, deformation with real ramification.

On $X_{0}$ the singularity (again denoted by $P$ ) is an isolated real point. Locally at $P$ this situation can be described inside $\mathbb{C}^{2}$ such that the curve has equation $x^{2}+y^{2}=0$ and the morphism is locally defined by $(x, y) \rightarrow x$ with coordinates compatible with the real structure on $X_{0}$. We use $U$ and $V$ as in Construction I. Consider the local deformation of $X_{0}$ at $P$ defined by $x^{2}+y^{2}=t$ with $t \in \mathbb{R}\left(|t|\right.$ very small). A point $Q \in U_{0} \backslash V_{0}$ has coordinates $(x, i x)$ or $(x,-i x)$ and $\sigma(Q)$ corresponds to resp. $(\bar{x},-i \bar{x})$ or $(\bar{x}, i \bar{x})$. We identify $(x, i x)$ (resp. $(x,-i x)))$ on $U_{0} \backslash V_{0}$ with $\left(x, i x \sqrt{-x^{2}+t}\right)$ (resp. $\left.\left(x,-{ }_{i x} \sqrt{-x^{2}+t}\right)\right)$ on $U_{t}$. This defines a Riemann surface $X_{t}(\mathbb{C})$. On $U_{t}$ we define $\sigma_{t}(x, y)=(\bar{x}, \bar{y})$ and we check that it behaves well under the identification. Consider $Q=\left(x,{ }_{i x} \sqrt{-x^{2}+t}\right)$ on $U_{t} \backslash V_{t}$. Since ${ }_{i x} \sqrt{-x^{2}+t}$ is close to $i x$ one has $\overline{i_{i x} \sqrt{-x^{2}+t}}$ is close to $-i \bar{x}$, hence

$$
\overline{i_{x} \sqrt{-x^{2}+t}}=-{ }_{i \bar{x}} \sqrt{-\bar{x}^{2}+t} .
$$

Hence $\sigma_{t}(Q)$ is identified with $(\bar{x},-i \bar{x})=\sigma_{0}(x, i x)$. We obtain a smooth real curve $X_{t}$ of genus $g+1$ together with a morphism $\pi_{t}: X_{t} \rightarrow \mathbb{P}^{1}$ of degree $k$ defined over $\mathbb{R}$.

In case $t>0$ there is a new component $C_{t}$ of $X_{t}(\mathbb{R})$ close to $P$ and under $\pi_{t}$ this maps to the interval $[-\sqrt{t}, \sqrt{t}]$. In particular $\delta_{C_{t}}\left(\pi_{t}\right)=0$. We call this the deformation with real ramification. In this case $X_{t}(\mathbb{C}) \backslash X_{t}(\mathbb{R})$ is connected if and only if $X(\mathbb{C}) \backslash X(\mathbb{R})$ is connected (see Figure 5).

In case $t<0$ then $X_{t}(\mathbb{R})$ contains no real point close to $P$. We call this the deformation without real ramification. In this case $X_{t}(\mathbb{C}) \backslash X_{t}(\mathbb{R})$ is always connected (see Figure 6). 


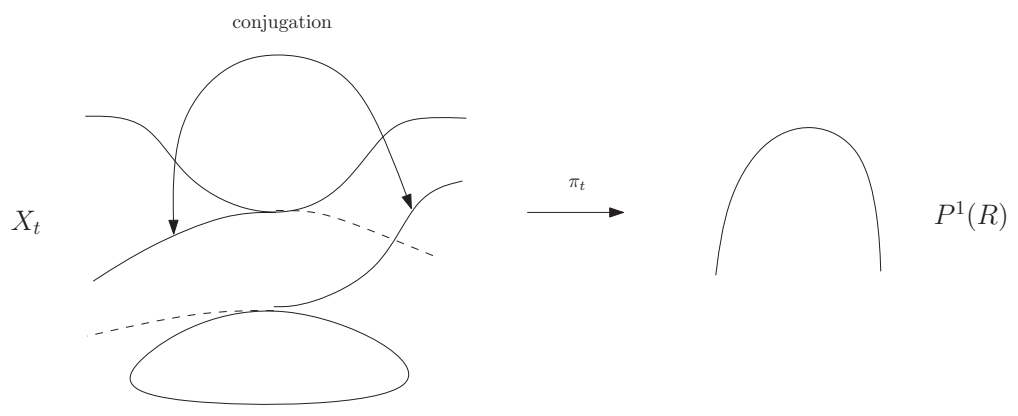

Fig. 6 Construction II, deformation without real ramification.

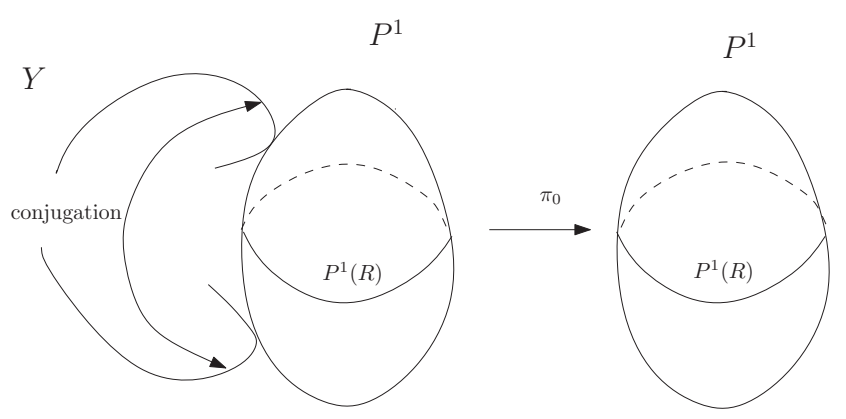

Fig. 7 Construction III, the curve $X_{0}$.

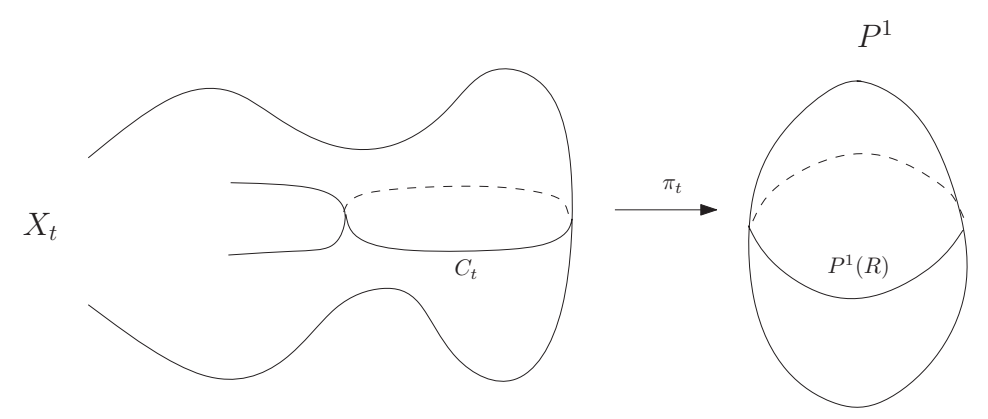

Fig. 8 Construction III, deformation.

\subsection{Construction III}

Let $Y$ be a real curve of genus $g$ and let $\pi: Y \rightarrow \mathbb{P}^{1}$ be a morphism of degree $k$ defined over $\mathbb{R}$. Assume $P+\bar{P}$ is a non-real point of $Y$ such that $\pi(P) \neq \pi(\bar{P})$ and $P, \bar{P}$ are not ramification points of $\pi_{\mathbb{C}}$. Now we let $X_{0}=Y \cup_{P+\bar{P}} \mathbb{P}^{1}$ be the union of $Y$ and $\mathbb{P}^{1}$ identifying $P$ with $\pi(P)$ (still denoted by $P$ ) and $\bar{P}$ with $\pi(\bar{P})$ (still denoted by $\bar{P}$ ). This singular curve has a natural morphism $\pi_{0}$ defined over $\mathbb{R}$ of degree $k+1$ to $\mathbb{P}^{1}$ having restriction $\pi$ to $Y$ and the identity to $\mathbb{P}^{1}$ (see Figure 7 ).

Choose a local deformation over $\mathbb{C}$ making it smooth at $P$ and take the complex conjugated local deformation at $\bar{P}$ and glue it with the remaining part of $X_{0}$. We obtain a smooth real curve $X_{t}$ of genus $g+1$ together with a morphism $\pi_{t}: X_{t} \rightarrow \mathbb{P}^{1}$ of degree $k+1$ defined over $\mathbb{R}$. One has $g\left(X_{t}\right)=g+1$ and $\mathbb{P}^{1}(\mathbb{R})$ on $X \cup_{P+} \mathbb{P}^{1}$ deforms to a connected component $C_{t}$ of $X_{t}(\mathbb{R})$ such that $\delta_{C_{t}}\left(\pi_{t}\right)=1$. For this construction $X_{t}(\mathbb{C}) \backslash X_{t}(\mathbb{R})$ is connected if and only if $X(\mathbb{C}) \backslash X(\mathbb{R})$ is connected (see Figure 8). 


\subsection{Construction IV}

Consider a double covering $\tau_{0}: R_{0} \rightarrow \mathbb{P}^{1}$ defined over $\mathbb{R}$. Assume $Y$ is a real curve without real points and $\pi: Y \rightarrow \mathbb{P}^{1}$ is a morphism of degree $k$ defined over $\mathbb{R}$ (in particular $k$ is even). Choose $P+\bar{P}$ on $\mathbb{P}^{1}$ (a non-real point) such that $P, \bar{P}$ are not branch points of $\pi_{\mathbb{C}}$ and choose non-real points $P_{0}+\overline{P_{0}}$ on $R_{0}$ and $Q+\bar{Q}$ on $Y$ such that $\tau_{0}(\mathbb{C})\left(P_{0}\right)=\pi(\mathbb{C})(Q)=P$. Consider the nodal curve $X_{0}=Y \cup_{P+\bar{P}} R_{0}$ obtained by identifying $P_{0}$ to $Q$ (still denoted by $Q$ ) and $\overline{P_{0}}$ to $\bar{Q}$ (still denoted by $\bar{Q}$ ). This is a singular curve defined over $\mathbb{R}$ without real points and $\pi$ together with $\tau_{0}$ induce a morphism of that singular curve to $\mathbb{P}^{1}$ defined over $\mathbb{R}$. Take a local deformation at $Q$ over $\mathbb{C}$ smoothing the singularity, take the complex conjugated deformation at $\bar{Q}$ and glue it with the remaining part of $X_{0}$ to obtain a smooth real curve $X_{t}$ of genus $g+1$ together with a morphism $\pi_{t}: X_{t} \rightarrow \mathbb{P}^{1}$ of degree $k+2$. This smooth real curve does not have any real point.

\subsection{Construction V}

Assume $Y$ is a real curve of genus $g$ without real points and assume $\pi: Y \rightarrow R_{0}$ is a morphism of degree $k$ defined over $\mathbb{R}$. Choose a non-real point $P_{0}+\overline{P_{0}}$ on $R_{0}$ such that $P_{0}, \overline{P_{0}}$ are not branch points of $\pi_{\mathbb{C}}$ and a nonreal point $P+\bar{P}$ on $Y$ with $\pi(P)=P_{0}$. Consider the nodal curve $X_{0}=Y \cup_{P+P_{0}} R_{0}$ obtained by identifying $P_{0}$ to $P$ (still denoted by $P$ ) and $\overline{P_{0}}$ to $\bar{P}$ (still denoted by $\bar{P}$ ). This is a singular curve defined over $\mathbb{R}$ without real points and $\pi$ together with the identity $1_{R_{0}}$ induces a morphism of degree $k+1$ of that singular curve to $R_{0}$ defined over $\mathbb{R}$. Take a local deformation at $P$ over $\mathbb{C}$ smoothing the singularity, take the complex conjugated deformation at $\bar{P}$ and glue it with the remaining part of $X_{0}$ to obtain a smooth real curve $X_{t}$ of genus $g+1$ together with a morphism $\pi_{t}: X_{t} \rightarrow R_{0}$ of degree $k+1$.

\section{Existence of real curves having pencils with prescribed topological proper- ties}

Let $X$ be a real curve of topological type $(g, s, a)$ and let $\pi: X \rightarrow \mathbb{P}^{1}$ be a morphism of degree $k$ defined over $\mathbb{R}$. Assume $s \geq 1$ and let $\underline{\delta}=\left(\delta_{1}, \ldots, \delta_{s}\right)$ be the topological degree of $\pi$. Of course we need $\sum_{i=1}^{s} \delta_{i} \leq k$. For each $s \in \mathbb{P}^{1}(\mathbb{R})$ the number of real points in $\pi^{-1}(s)$ counted with multiplicity has the same parity as $\sum_{i=1}^{s} \delta_{i}$. Since the non-real points of $X$ are pairs of conjugated points on $X(\mathbb{C})$ it follows $k-\sum_{i=1}^{s} \delta_{i} \equiv 0(\bmod 2)$. In case $\sum_{i=1}^{s} \delta_{i}=k$ then for each $s \in \mathbb{P}^{1}(\mathbb{R})$ the fiber $\pi^{-1}(s)$ has exactly $\delta_{i}$ different points on $C_{i}$. Since some fiber needs to contain points of $C_{s}$ one has $\delta_{s} \geq 1$ in that case, hence $\sum_{i=1}^{s} \delta_{i} \leq k-2$ in case $\delta_{s}=0$ (see also [8]).

Definition 3.1 We say $\delta_{1} \geq \cdots \geq \delta_{s} \geq 0$ is an admissible topological degree for morphisms of degree $k$ if

- $\sum_{i=1}^{s} \delta_{i} \leq k$,

- $k-\sum_{i=1}^{s} \delta_{i} \equiv 0(\bmod 2)$,

- $\sum_{i=1}^{s} \delta_{i} \leq k-2$ in case $\delta_{s}=0$.

In case $s=0$ then we consider $\underline{\delta}$ being empty and the only condition becomes $k \equiv 0(\bmod 2)$. For the next theorem we need some more restriction. In case there is a morphism $\pi: X \rightarrow \mathbb{P}^{1}$ of degree $k$ with $\sum_{i=1}^{s} \delta_{i}=k$ then by definition $\pi^{-1}\left(\mathbb{P}^{1}(\mathbb{R})\right)=X(\mathbb{R})$, hence $X(\mathbb{C}) \backslash X(\mathbb{R})=\pi^{-1}\left(\mathbb{P}^{1}(\mathbb{C}) \backslash \mathbb{P}^{1}(\mathbb{R})\right)$. Since $\mathbb{P}^{1}(\mathbb{C}) \backslash \mathbb{P}^{1}(\mathbb{R})$ is disconnected it follows $X(\mathbb{C}) \backslash X(\mathbb{R})$ is disconnected too, hence $a(X)=0$. Therefore, in case $a(X)=1$ one always has $\sum_{i=1}^{s} \delta_{i} \leq k-2$.

Theorem 3.2 Let $k \geq 3$, let $(g, s, a)$ be an admissible topological type of real curves and let $\underline{\delta}$ be an admissible topological degree for morphisms of degree $k$. Assume $\sum_{i=1}^{s} \delta_{i} \leq k-2$ in case $a=1$. There exists a real curve $X$ of topological type $(g, s, a)$ having a morphism $\pi: X \rightarrow \mathbb{P}^{1}$ of degree $k$ of topological degree $\underline{\delta}$.

Remark 3.3 The case $k=2$ is contained in e.g. [14, Section 6]. In that case there are more restrictions in the case $a=0$. Nevertheless, the existence of real hyperelliptic curves is the starting point in a lot of cases of the proof of the theorem, even in Case 5 (see 3.2.5) which is excluded for real hyperelliptic curves unless $s=g+1$.

Section 3.1 is the proof of Theorem 3.2 in case $a=1$ and Section 3.2 is the proof of Theorem 3.2 in case $a=0$. In Section 3.3 we prove the corresponding theorem for morphisms to $R_{0}$. 


\subsection{The case $a=1$}

First we consider the case $s \neq 0$. Let $s^{\prime}$ be the number of $\delta_{i} \neq 0$ and let $\widetilde{X}$ be a real hyperelliptic curve of topological type $\left(g-s^{\prime}, s-s^{\prime}, 1\right)$ (such curves do exist, see [14, Section 6]). Let $\widetilde{\pi}$ be the associated double covering of $\mathbb{P}^{1}$ then it follows that $\delta_{\widetilde{C}_{i}}(\widetilde{\pi})=0$ for $1 \leq i \leq s-s^{\prime}$. Applying Construction III $s^{\prime}$ times we obtain $\pi_{1}: X_{1} \rightarrow \mathbb{P}^{1}$ with $\operatorname{deg}\left(\pi_{1}\right)=2+s^{\prime} ; g\left(X_{1}\right)=g$ and $X_{1}(\mathbb{R})$ has $s$ components $C_{1}^{1}, \ldots, C_{s}^{1}$ such that

$$
\delta_{C_{i}^{1}}\left(\pi_{1}\right)= \begin{cases}1 & \text { for } \quad 1 \leq i \leq s^{\prime}, \\ 0 & \text { for } \quad s^{\prime}+1 \leq i \leq s,\end{cases}
$$

in particular $X_{1}$ has topological type $(g, s, 1)$. For each $1 \leq i \leq s^{\prime}$ we use Construction I $\delta_{i}-1$ times on $C_{i}^{1}$ and using the deformation without real ramification. We obtain $\pi_{2}: X_{2} \rightarrow \mathbb{P}^{1}$ with $\operatorname{deg}\left(\pi_{2}\right)=2+\sum_{i=1}^{s^{\prime}} \delta_{i}$, $g\left(X_{2}\right)=g$ and $X_{2}(\mathbb{R})$ has $s$ components $C_{1}^{2}, \ldots, C_{s}^{2}$ such that

$$
\delta_{C_{i}^{2}}\left(\pi_{2}\right)= \begin{cases}\delta_{i} & \text { for } \quad 1 \leq i \leq s^{\prime} \\ 0 & \text { for } \quad s^{\prime}+1 \leq i \leq s\end{cases}
$$

hence $\pi_{2}$ has topological degrees $\underline{\delta}$ and $X_{2}$ has topological type $(g, s, 1)$. In case $s \neq s^{\prime}$ we apply Construction I $k-2-\sum_{i=1}^{s^{\prime}} \delta_{i}$ times on $C_{s^{\prime}+1}^{2}$ using the deformation with real ramification. In case $s=s^{\prime}$ and $s^{\prime} \neq 0$ we apply Construction I $k-2-\sum_{i=1}^{s^{\prime}} \delta_{i}$ times on $C_{1}^{2}$ alternating the deformation with and without real ramification. We obtain a real curve $X$ of topological type $(g, s, 1)$ and a real morphism $\pi: X \rightarrow \mathbb{P}^{1}$ having degree $k$ and topological degree $\underline{\delta}$. Indeed, in this final step the degrees of the restrictions of $\pi$ do not change since $k-2-$ $\sum_{i=1}^{s^{\prime}} \delta_{i}$ is even.

Now assume $s=0$ (the case of no real points). In this case $k$ is even. In case $g<k$ a general divisor $D$ on a real curve $X$ of degree $k$ gives rise to a base point free linear system $|D|$. A general pencil in it defined over $\mathbb{R}$ gives rise to a real base point free $g_{k}^{1}$ on $X$, hence to a real morphism $\pi: X \rightarrow \mathbb{P}^{1}$ of degree $k$. So we can assume $g \geq k$. Start with a hyperelliptic curve $\widetilde{X}$ of genus $g-(k / 2)+1$ with $\widetilde{X}(\mathbb{R})=\emptyset$. We apply Construction IV $(k / 2)-1$ times. Again we obtain a real curve $X$ of genus $g$ with $X(\mathbb{R})=\emptyset$ and having a real morphism $\pi: X \rightarrow \mathbb{P}^{1}$ of degree $k$.

\subsection{The case $a=0$}

Again, let $s^{\prime}$ be the number of $\delta_{i} \neq 0$.

\subsubsection{Case 1}

Assume $s^{\prime}=s=1$ and $\delta_{1}=k$. In particular $g \equiv 0(\bmod 2)$. Let $X_{0}$ be a real hyperelliptic curve of genus $g$ having $a\left(X_{0}\right)=0, s\left(X_{0}\right)=1$, hence for the hyperelliptic real morphism $\pi_{0}: X_{0} \rightarrow \mathbb{P}^{1}$ the unique component $C_{1}$ of $X_{0}(\mathbb{R})$ has topological degree 2 (see [14, Section 6]). Apply $k-2$ times Construction I using the deformation without real ramification. We obtain a real curve $X$ of topological type $(g, 1,0)$ such that $X$ has a covering $\pi: X \rightarrow \mathbb{P}^{1}$ defined over $\mathbb{R}$ of degree $k$ such that $\delta_{C_{1}}(\pi)=k$.

\subsubsection{Case 2}

Assume $s>1$ and $\sum_{i=1}^{s} \delta_{i}=k$ in which case $s^{\prime}=s$. In case all $\delta_{i}=1$ then $s=k$ and therefore $g \equiv k+1$ (mod 2). In particular $g-k+2$ is odd. Since $k=s$ and $s \leq g+1$ also $g-k+2 \geq 1$. Let $X_{0}$ be a real hyperelliptic curve of genus $g_{0}=g-k+2$ having $a\left(X_{0}\right)=0, s\left(X_{0}\right)=2$ (see [14, Section 6]). Hence, for the real hyperelliptic morphism $\pi_{0}: X_{0} \rightarrow \mathbb{P}^{1}$ the 2 components $C$ of $X_{0}(\mathbb{R})$ satisfy $\delta_{C}\left(\pi_{0}\right)=1$. Apply $k-2$ times Construction III. We obtain a real curve $X$ of genus $g$ having $a(X)=0, s(X)=k$ such that $X$ has a covering $\pi: X \rightarrow \mathbb{P}^{1}$ defined over $\mathbb{R}$ of degree $k$ such that each component $C$ of $X(\mathbb{R})$ satisfies $\delta_{C}(\pi)=1$.

Assume not all $\delta_{i}=1$, hence $\delta_{1} \geq 2$. Let $X_{0}$ be a real hyperelliptic curve of genus $g_{0}=g-s+1$ (in particular $g_{0}$ is even) as in Case 1. Apply Construction III $s-1$ times. We obtain a real curve $X_{1}$ of genus $g$ having $a(X)=0, s(X)=s$ such that $X_{1}$ has a covering $\pi_{1}: X_{1} \rightarrow \mathbb{P}^{1}$ defined over $\mathbb{R}$ of degree $s+1$ such 
that exactly one component $C_{1}$ of $X_{1}(\mathbb{R})$ satisfies $\delta_{C_{1}}\left(\pi_{1}\right)=2$ while the other components $C_{2}, \ldots, C_{s}$ satisfy $\delta_{C_{i}}\left(\pi_{1}\right)=1$. We apply Construction I $\delta_{1}-2$ times without real ramification starting with $C_{1}$ and for $2 \leq i \leq s$ we apply Construction $\mathrm{I} \delta_{i}-1$ times without real ramification starting with $C_{i}$. We obtain a real curve $X$ of genus $g$ satisfying $a(X)=0, s(X)=s$ such that $X$ has a covering $\pi: X \rightarrow \mathbb{P}^{1}$ defined over $\mathbb{R}$ of degree

$$
s+1+\left(\delta_{1}-2\right)+\sum_{i=2}^{s}\left(\delta_{i}-1\right)=\sum_{i=1}^{s} \delta_{i}=k
$$

having topological degree $\underline{\delta}$.

\subsubsection{Case 3}

Assume $\sum_{i=1}^{s^{\prime}} \delta_{i}<k$ and $s^{\prime}=s$. It follows that $s^{\prime} \geq 1$ and $g \equiv s^{\prime}+1(\bmod 2)$. In particular $g-s^{\prime}+1$ is even. Let $X_{0}$ be a real hyperelliptic curve of genus $g-s^{\prime}+1$ such that $a\left(X_{0}\right)=0, s\left(X_{0}\right)=1$ and for the real hyperelliptic morphism $\pi_{0}: X_{0} \rightarrow \mathbb{P}^{1}$ one has that the unique component $C_{1}$ of $X_{0}(\mathbb{R})$ satisfies $\delta_{C_{1}}\left(\pi_{0}\right)=2$ (see [14, Section 6]). Apply Construction I using the deformation with real ramification to $C_{1}$ and apply Construction III $s^{\prime}-1$ times. We obtain a real curve $X_{1}$ of genus $g$ having $a\left(X_{1}\right)=0, s\left(X_{1}\right)=s^{\prime}=s$ such that there is a morphism $\pi_{1}: X_{1} \rightarrow \mathbb{P}^{1}$ of degree $s^{\prime}+2$ defined over $\mathbb{R}$ such that each component $C_{i}$ of $X_{1}(\mathbb{R})$ satisfies $\delta_{C_{i}}\left(\pi_{1}\right)=1$.

For $1 \leq i \leq s^{\prime}$ apply Construction $\mathrm{I} \delta_{i}-1$ times using the deformation without real ramification starting with $C_{i}$. We obtain a real curve $X_{2}$ of genus $g$ having $a\left(X_{2}\right)=0, s\left(X_{2}\right)=s^{\prime}=s$ and such that there is a morphism $\pi_{2}: X_{2} \rightarrow \mathbb{P}^{1}$ of degree

$$
s^{\prime}+2+\left(\sum_{i=1}^{s} \delta_{i}\right)-s^{\prime}=\left(\sum_{i=1}^{s} \delta_{i}\right)+2
$$

of topological degree $\underline{\delta}$.

By assumption $k-\left(\sum_{i=1}^{s} \delta_{i}\right)-2$ is an even non-negative integer. Apply Construction $\mathrm{I} k-\left(\sum_{i=1}^{s} \delta_{i}\right)-2$ times starting with $C_{1}$ alternating deformation with and without real ramification (starting with real ramification) we end up with a real curve $X$ of genus $g$ satisfying $a(X)=0, s(X)=s^{\prime}=s$ and such that there is a morphism $\pi: X \rightarrow \mathbb{P}^{1}$ of degree $k$ of topological degree $\underline{\delta}$.

\subsubsection{Case 4}

Assume $\sum_{i=1}^{s^{\prime}} \delta_{i}<k, s>s^{\prime}$ and $s^{\prime} \geq 1$. We start using the construction of Case 3 making a real curve $X^{\prime}$ of genus $g-s+s^{\prime}$ satisfying $a\left(X^{\prime}\right)=0, s\left(X^{\prime}\right)=s^{\prime}$ and such that there is a real morphism $\pi^{\prime}: X^{\prime} \rightarrow \mathbb{P}^{1}$ of degree $k$ such that, for the components $C_{1}, \ldots, C_{s^{\prime}}$ one has $\delta_{C_{i}}\left(\pi^{\prime}\right)=\delta_{i}$. Notice that, since $g-s \equiv 1(\bmod 2)$ one has $g-s+s^{\prime} \equiv s^{\prime}+1(\bmod 2)$. Since $\sum_{i=1}^{s^{\prime}} \delta_{i}<k$ the set $X^{\prime}(\mathbb{R}) \backslash \pi^{\prime-1}\left(\mathbb{P}^{1}(\mathbb{R})\right)$ is not empty. Therefore we can apply Construction II $s-s^{\prime}$ times using a deformation with real ramification. We obtain a real curve $X$ of genus $g$ such that $a(X)=0, s(X)=s$ and $X$ has a real covering $\pi: X \rightarrow \mathbb{P}^{1}$ of degree $k$ of topological degree $\underline{\delta}$.

\subsubsection{Case 5}

Assume $s^{\prime}=0$. In this case $k$ is even. Let $X_{0}$ be a hyperelliptic curve of genus $g-s+1$ (hence of even genus) such that $a\left(X_{0}\right)=0, s\left(X_{0}\right)=1$ and if $\pi_{0}: X_{0} \rightarrow \mathbb{P}^{1}$ is the hyperelliptic morphism then the unique component $C_{1}$ of $X_{0}(\mathbb{R})$ satisfies $\delta_{C_{1}}\left(\pi_{0}\right)=2$ (see [14, Section 6]). Apply Construction I $k-2$ times using the deformation with real ramification. One obtains a real curve $X_{1}$ of genus $g-s+1$ such that $a\left(X_{1}\right)=0$ and $s\left(X_{1}\right)=1$ and a morphism $\pi_{1}: X_{1} \rightarrow \mathbb{P}^{1}$ of degree $k$ such that for the unique component $C_{1}$ of $X_{1}(\mathbb{R})$ one has $\delta_{C_{1}}\left(\pi_{1}\right)=0$. It follows that $X_{1}(\mathbb{R}) \backslash\left(\pi_{1}\right)^{-1}\left(\mathbb{P}^{1}(\mathbb{R})\right) \neq \emptyset$ and so we apply $s-1$ times Construction II with real ramification. We obtain a real curve $X$ of genus $g$ such that $a(X)=0, s(X)=s$ and there exists a real morphism $\pi: X \rightarrow \mathbb{P}^{1}$ of degree $k$ such that for each component $C$ of $X(\mathbb{R})$ one has $\delta_{C}(\pi)=0$.

\subsection{Morphisms to $R_{0}$}

Assume $X$ is a real curve of genus $g$ with $X(\mathbb{R})=\emptyset$ and assume $\pi: X \rightarrow R_{0}$ is a morphism of degree $k$ defined over $\mathbb{R}$. Consider the associated morphism of Riemann surfaces $\pi(\mathbb{C}): X(\mathbb{C}) \rightarrow R_{0}(\mathbb{C})=\mathbb{P}^{1}(\mathbb{C})$ and let $|D|$ be 
the associated complete linear system on $X(\mathbb{C})$. We are going to show that $|D|$ is not induced by a linear system on $X$ (i.e. $|D|$ does not contain a real divisor).

Notice that complex conjugation of divisors on $X(\mathbb{C})$ acts on $|D|$. Indeed, choose a non-real point $Q+\bar{Q}$ on $R_{0}$ and let $\pi(\mathbb{C})^{-1}(Q)=D_{1}$. Since $\pi(\mathbb{C})^{-1}(\bar{Q})=\overline{D_{1}}$ one has $D_{1} \sim \overline{D_{1}}$. For any $E \in|D|$ one has $E \sim D_{1}$. This implies $\bar{E} \sim \overline{D_{1}}$ hence also $E \sim \bar{E}$. Now $\pi(\mathbb{C})$ corresponds to a line $L$ in $|D|$ that is invariant under this complex conjugation. If $|D|$ would be real it would imply $L$ is a real line, hence containing a real point. Such real point corresponds to a real divisor and this would be a fiber of $\pi(\mathbb{C})$ invariant under complex conjugation but such fibers do not exist.

This implies $\mathcal{O}_{X}(D)$ corresponds to a point of $\operatorname{Pic}^{k}(X)(\mathbb{R}) \backslash \operatorname{Pic}^{k}(X)(\mathbb{R})^{+}$, hence $k \equiv g+1$ (mod 2) (see [14]). In this section we consider pencils corresponding to points in $\operatorname{Pic}^{k}(X)(\mathbb{R}) \backslash \operatorname{Pic}^{k}(X)(\mathbb{R})^{+}$(in particular $k \equiv g+1(\bmod 2))$.

Theorem 3.4 Let $g$ and $k \equiv g+1(\bmod 2)$ be nonnegative integers. In case $k \geq g+1$ and $X$ is a real curve of genus $g$ with $X(\mathbb{R})=\emptyset$ then there exists a morphism $\pi: X \rightarrow R_{0}$ of degree $k$. In case $k<g+1$ then there exists a real curve of genus $g$ with $X(\mathbb{R})=\emptyset$ having a morphism $\pi: X \rightarrow R_{0}$ of degree $k$.

Proof. First assume $k \geq g+1$. Choose an effective divisor $D$ such that $\mathcal{O}_{X}(D)$ corresponds to a point on $\operatorname{Pic}^{k}(X)(\mathbb{R}) \backslash \operatorname{Pic}^{k}(X)(\mathbb{R})^{+}$. Then $|D|$ is not defined over $\mathbb{R}$ but complex conjugation of divisors is defined on $|D|$. Choose $D_{1} \in|D|$ and let $L$ be the line in $|D|$ connecting $D_{1}$ to $\overline{D_{1}}$. This corresponds to a morphism $\pi(\mathbb{C}): X(\mathbb{C}) \rightarrow \mathbb{P}^{1}(\mathbb{C})$. Since $L=\bar{L} \subset|D|$ it follows $\pi(\mathbb{C})$ is equivariant using the complex conjugation on $X(\mathbb{C})$ and some antiholomorphic involution on $\mathbb{P}^{1}(\mathbb{C})$ without fixed points. This implies $\pi(\mathbb{C})$ is induced by a morphism $\pi: X \rightarrow R_{0}$ defined over $\mathbb{R}$.

Next assume $k \leq g+1$ and $k \equiv g+1(\bmod 2)$. Let $X_{1}$ be a real curve of genus $g-k+2$ having a morphism $f_{1}: X_{1} \rightarrow R_{0}$ of degree 2 (such curves exist since $g-k+2$ is odd, see [14, Section 6]). Then use Construction $\mathrm{V} k-2$ times .

Remark 3.5 In his paper [6] the author obtains Theorem 3.4 by fixing a real divisor $D$ on $R_{0}$ and using the Riemann Existence Theorem using coverings on $R_{0}(\mathbb{C}) \backslash D$. Although the author gives no argument for the existence of an antiholomorphic involution on a suited choice of such a covering this reasoning can be applied using Klein surfaces (see [1] for the foundations of that theory). Also in that paper he obtains Theorem 3.2 for the case $s=0$ but in that argument details are omitted.

In his paper [7] the author also gives an argument for the existence of real curves $X$ of given topological type having a morphism $\pi: X \rightarrow \mathbb{P}^{1}$ of degree $k$. For the case $a(X)=0$ he uses deformations of hyperelliptic and trigonal curves. The case $a(X)=1$ is contained in [5]. In that paper the author uses deformations of singular real curves on suited real rational surfaces using Brusotti-type arguments. We use arguments directly applied to coverings which is more natural and easier. In both cases it is not clear to us how to obtain the existence of all admissible topological degrees for $\pi$ using those deformations. We also give some remarks on some of the arguments used in those papers (especially [7]) in Remark 4.6.

In $[14$, Section 8$]$ one considers real trigonal curves and one asks about determining the topological types of such curves. In Theorem 3.2 we obtain that all admissible topological types can be realized as real trigonal curves. A (in principle similar) proof is described in [7, Proposition 2.1]. A different proof using Fuchsian and NEC (non-euclidean crystallographic) groups (and obtaining much more detailled information concerning the case of cyclic trigonal coverings) is given in [10].

\section{Some Brill-Noether Theory for real pencils on real curves}

On $\operatorname{Pic}^{k}(X)(\mathbb{C})$ we consider the closed subspace $W_{k}^{1}(X)$ representing invertible sheafs on $X_{\mathbb{C}}$ satisfying $h^{0}(L) \geq 2$. This subspace $W_{k}^{1}(X)$ is invariant under conjugation, hence it is defined over $\mathbb{R}$. We write $W_{k}^{1}(X)(\mathbb{R})$ to denote its set of real points and $W_{k}^{1}(X)(\mathbb{R})^{+}=W_{k}^{1}(X)(\mathbb{R}) \cap \operatorname{Pic}^{k}(X)(\mathbb{R})^{+}$. In case $k \geq g+1$ one has $W_{k}^{1}(X)=\operatorname{Pic}^{k}(X)(\mathbb{C})$, so we assume from now on that $k \leq g$.

The study of those spaces $W_{k}^{1}(X)$ for general complex curves $X$ of genus $g$ is the Brill-Noether Theory for pencils. The main results are the following. First consider the Brill-Noether number $\rho_{k}^{1}(g)=2 k-g-2$. For a general complex curve $X$ one has $W_{k}^{1}(X)=\emptyset$ in case $\rho_{k}^{1}(g)<0$ and $\operatorname{dim}\left(W_{k}^{1}(X)\right)=\rho_{k}^{1}(g)$ in case $\rho_{k}^{1}(g) \geq 0$. 
Also in case $\rho_{k}^{1}(g) \geq 0$ then $W_{k}^{1}(X) \neq \emptyset$ for all complex curves $X$. Here "general" means: the statement holds for all curves $X$ on a dense open subset of the moduli space $M_{g}$ of curves of genus $g$.

In [9] one considers Brill-Noether theory of $W_{k}^{1}(X)(\mathbb{R})$ for real curves $X$. There are important differences with the complex case. We consider the moduli space $M_{g / \mathbb{R}}$ of real curves of genus $g$ representing the isomorphism classes over $\mathbb{R}$ of real curves of genus $g$. We recall some facts on it (for details see [19]). It is a semi-analytic real variety of dimension $3 g-3$ (as a matter of fact it has a non-empty boundary, see [18] for its description). For each topological type $(g, s, a)$ there is a unique connected component $M_{g / \mathbb{R}}(g, s, a)$ of $M_{g / \mathbb{R}}$. This connected component is the quotient of a Teichmüller space (which is a connected real analytic manifold) by means of a discontinuous action of a modular group. Inside such component $M_{g / \mathbb{R}}(g, s, a)$, curves having a certain type of real linear system give rise to semi-analytic subvarieties of $M_{g / \mathbb{R}}(g, s, a)$. Therefore there is in general no description of behavior on a dense open subset of $M_{g / \mathbb{R}}(g, s, a)$ and we need to modify the meaning of "general".

Definition 4.1 Let $\mathrm{P}$ be some property concerning real linear systems on real curves. We say there is a general real curve of topological type $(g, s, a)$ satisfying property $\mathrm{P}$ if there exists a non-empty open subset $U$ of $M_{g / \mathbb{R}}(g, s, a)$ such that $\mathrm{P}$ holds for all curves corresponding to a point of $U$.

Example 4.2 To illustrate the difference with the complex situation we consider $W_{3}^{1}$ for curves of genus 4 $\left(\rho_{3}^{1}(4)=0 \geq 0\right)$ (see [14, Section 8]). A real curve of type $(4,0,1)$ has no real $g_{3}^{1}$ since all its real divisors have even degree. In case $s \neq 0$ there exists a general real curve of type $(4, s, a)$ having a real $g_{3}^{1}$. In case $(s, a) \neq(1,0)$ there also exists a general real curve of type $(4, s, a)$ having no real $g_{3}^{1}$. In Proposition 4.7, as an application of Theorem 3.2, we give a description of the situation in case $(s, a)=(1,0)$.

In [9] it is proved that in case $\rho_{k}^{1}(g) \geq 0$ and $s \neq 0$ then there is a general real curve of type $(g, s, a)$ such that $W_{k}^{1}(X)(\mathbb{R}) \neq \emptyset$. In order to prove this result the author constructs a real compactification of the real Hurwitz space of coverings of degree $k$. The restriction $s \neq 0$ has a technical cause. Working with families of real curves the author needs the existence of a section defined over $\mathbb{R}$. In particular such a section guaranties that the relative Picard scheme of such families represents the Picard functor (see [16]).

Now we are going to use the existence results from Section 3 to give another proof. In that way we obtain a finer statement involving the covering degree. In the argument we make intensive use of Brill-Noether theory for complex curves and we work with the space of morphisms to $\mathbb{P}^{1}$ instead of the Hurwitz space. This space of morphisms is defined using Hilbert schemes in [15, Section 4.c]. In particular we do not need relative Picard schemes to finish our arguments, hence we do not need the restriction $s \neq 0$. In case $s=0$ we consider both $W_{k}^{1}(X)(\mathbb{R})^{+}$and $W_{k}^{1}(X)(\mathbb{R}) \backslash W_{k}^{1}(X)(\mathbb{R})^{+}$.

In case $f: X \rightarrow R_{0}$ is a morphism of degree $k$ then $f_{\mathbb{C}}: X_{\mathbb{C}} \rightarrow \mathbb{P}_{\mathbb{C}}^{1}$ corresponds to a linear system $g_{k}^{1} \subset|L|$ for some $L \in W_{k}^{1}(X)(\mathbb{R}) \backslash W_{k}^{1}(X)(\mathbb{R})^{+}$. In order to study $W_{k}^{1}(X)(\mathbb{R}) \backslash W_{k}^{1}(X)(\mathbb{R})^{+}$using morphims, we also need the converse statement.

Lemma 4.3 Let $L \in W_{k}^{1}(X)(\mathbb{R}) \backslash W_{k}^{1}(X)(\mathbb{R})^{+}$and assume $|L|$ is base point free of dimension 1 . Then there is a morphism $f: X \rightarrow R_{0}$ of degree $k$ such that $f_{\mathbb{C}}$ corresponds to $|L|$.

Proof. See [12, Example 1].

Let $X$ be a real curve of topological type $(g, s, a)$ and let $g_{k}^{1}$ be a complete base point free linear system on $X$. We say $g_{k}^{1}$ has topological degree $\underline{\delta}$ if the morphisms $f: X \rightarrow \mathbb{P}^{1}$ associated to $g_{k}^{1}$ have topological degree $\underline{\delta}$.

Let ${ }^{\circ} W_{k}^{1}(X)$ be the subspace of $W_{k}^{1}(X)$ parameterizing complete base point free linear systems $g_{k}^{1}$ on $X_{\mathbb{C}}$. It is the complement of $W_{k-1}^{1}(X)+W_{1}^{0}(X)$ in $W_{k}^{1}(X)$, hence it is Zariski-open in $W_{k}^{1}(X)$. In particular ${ }^{\circ} W_{k}^{1}(X)(\mathbb{R})$ is Zariski-open in $W_{k}^{1}(X)(\mathbb{R})$. Because the topological degree is a discrete invariant it is constant on connected components of ${ }^{\circ} W_{k}^{1}(X)(\mathbb{R})$. Let ${ }^{\circ} W_{k}^{1}(X)(\underline{\delta})$ be the sublocus of ${ }^{\circ} W_{k}^{1}(X)(\mathbb{R})$ such that it parameterizes linear systems $g_{k}^{1}$ of topological degree $\underline{\delta}$ and let $W_{k}^{1}(X)(\underline{\delta})$ be its closure in $W_{k}^{1}(X)(\mathbb{R})$ (it is a union of irreducible components of $\left.W_{k}^{1}(X)(\mathbb{R})\right)$.

Theorem 4.4 Let $\underline{\delta}$ be an admissible topological degree of base point free linear systems $g_{k}^{1}$ on real curves of topological type $(g, s, a)$ such that $\sum_{i=1}^{s} \delta_{i} \leq k-2$ in case $a=1$.

(1) If $\rho_{k}^{1}(g)<0$ then there is no general real curve $X$ of type $(g, s, a)$ such that $W_{k}^{1}(X)(\underline{\delta})$ is not empty.

(2) If $\rho_{k}^{1}(g) \geq 0$ then there is a general real curve $X$ of topological type $(g, s, a)$ such that $W_{k}^{1}(X)(\underline{\delta})$ is non-empty and it is a real algebraic subset of $\operatorname{Pic}^{k}(X)(\mathbb{R})$ of dimension $\rho_{k}^{1}(g)$. 
(3) In case $s=0$ and $k \equiv g+1(\bmod 2)$ then in case $\rho_{k}^{1}(g)<0$ there is no general real curve $X$ of topological type $(g, 0,1)$ such that $W_{k}^{1}(X)(\mathbb{R}) \backslash W_{k}^{1}(X)(\mathbb{R})^{+}$is not empty. In case $\rho_{k}^{1}(g) \geq 0$ then there is a general real curve $X$ of topological type $(g, 0,1)$ such that $W_{k}^{1}(X)(\mathbb{R}) \backslash W_{k}^{1}(X)(\mathbb{R})^{+}$is not empty and has dimension $\rho_{k}^{1}(g)$.

In order to prove this theorem we are going to use spaces parameterizing morphisms to $\mathbb{P}^{1}$. First we introduce some definition that will be useful for the proof.

Definition 4.5 A suited family of curves of genus $g$ is a projective morphism $\pi: \mathcal{C} \rightarrow S$ such that

- $S$ is smooth, irreducible and quasi-projective,

- each fiber of $\pi$ is a smooth connected curve of genus $g$,

- for each $s \in S$ the Kodaira-Spencer map $T_{s}(S) \rightarrow H^{1}\left(\pi^{-1}(s), T_{\pi^{-1}(s)}\right)$ is bijective.

In case $X$ is a given smooth curve of genus $g$ then we say $\pi: \mathcal{C} \rightarrow S$ is a suited family for $X$ if moreover some fiber of $\pi$ is isomorphic to $X$. If moreover $X$ is defined over $\mathbb{R}$ then we also assume $\pi: \mathcal{C} \rightarrow S$ is defined over $\mathbb{R}$ and $X$ is isomorphic over $\mathbb{R}$ to some fiber of $\pi$ over a real point of $S$.

Such suited families do exist (see [12, Section 3], in that paper one considers real curves with real points and one needs the family to have a real section, in our situation one does not need to use $S^{\prime}$ from loc. cit.). From a suited family we obtain a morphism $S \rightarrow M_{g}$ and in case $K=\mathbb{R}$ we also obtain a morphism $S(\mathbb{R}) \rightarrow M_{g / \mathbb{R}}$. From the bijectivity of the Kodaira-Spencer map it follows those morphisms to the moduli space have finite fibers. Therefore it is enough to prove the statements of Theorem 4.4 for real curves corresponding to fibers of $\pi$ at general points of $S(\mathbb{R})$.

Let $\pi: \mathcal{C} \rightarrow S$ be a suited family of curves of genus $g$ defined over $K$ (being $\mathbb{C}$ or $\mathbb{R}$ ). For a noetherian $S$-scheme $T$ we consider the set $\mathcal{H}_{k}(\pi)(T)$ of finite $T$-morphisms $\mathcal{C} \times{ }_{S} T \rightarrow \mathbb{P}^{1} \times T$ of degree $k$. This functor is representable by an $S$-scheme $\pi_{k}: H_{k}(\pi) \rightarrow S$ (see [15, Section 4.c]). Let $f: X \rightarrow \mathbb{P}_{\mathbb{C}}^{1}$ be a morphism corresponding to a point $[f]$ on $H_{k}(\pi)(\mathbb{C})$ and consider the associated exact sequence

$$
0 \longrightarrow T_{X} \longrightarrow f^{*}\left(T_{\mathbb{P}^{1}}\right) \longrightarrow N_{f} \longrightarrow 0 .
$$

From Horikawa's deformation theory of holomorphic maps (see [17], see also [23, 3.4.2]) it follows $T_{[f]}\left(H_{k}(\pi)\right)$ is canonically identified with $H^{0}\left(X, N_{f}\right)$ and since $H^{1}\left(X, N_{f}\right)=0$ it follows $H_{k}(\pi)$ is smooth of dimension $2 k+2 g-2$. In case $K=\mathbb{R}$ it follows $H_{k}(\pi)(\mathbb{R})$ is a smooth real manifold of dimension $2 k+2 g-2$ (of course $H_{k}(\pi)(\mathbb{R})$ need not be connected).

In case $K=\mathbb{R}$ then for a noetherian $S$-scheme $T$ we consider the set $\mathcal{H}_{k}^{R}(\pi)(T)$ of finite $T$-morphisms $\mathcal{C} \times{ }_{S} T \rightarrow R_{0} \times T$ of degree $k$. This functor is represented by an $S$-scheme $\pi_{k}^{R}: H_{k}^{R}(\pi) \rightarrow S$ and using the same arguments we obtain $H_{k}^{R}(\pi)$ is smooth of dimension $2 k+2 g-2$ and $H_{k}^{R}(\pi)(\mathbb{R})$ is a smooth real manifold of dimension $2 k+2 g-2$ (again, it need not be connected).

Because $\operatorname{dim}\left(A u t\left(\mathbb{P}_{\mathbb{C}}^{1}\right)\right)=3$ the non-empty fibers of $\pi_{k}(\mathbb{C})\left(\right.$ and $\left.\pi_{k}^{R}(\mathbb{C})\right)$ do have dimension at least 3. Hence the image of $\pi_{k}(\mathbb{C})$ (and of $\pi_{k}^{R}(\mathbb{C})$ ) has dimension at most

$$
2 k+2 g-5=3 g-3+\rho_{k}^{1}(g) .
$$

Hence in case $\rho_{k}^{1}(g)<0$ then a general point of $S(\mathbb{C})$ does not belong to the image of $\pi_{k}(\mathbb{C})\left(\right.$ or $\left.\pi_{k}^{R}(\mathbb{C})\right)$. Now assume $\rho_{k}^{1}(g) \geq 0$. From the arguments in [24, Appendix] it follows that each component of $H_{k}(\pi)(\mathbb{C})$ (or $H_{k}^{R}(\pi)(\mathbb{C})$ ) dominates $S(\mathbb{C})$. Let $H_{k}^{\prime}(\pi)(\mathbb{C})\left(\right.$ resp. $H_{k}^{\prime R}(\pi)(\mathbb{C})$ ) be the closed subspace of $H_{k}(\pi)(\mathbb{C})$ (resp. $H_{k}^{R}(\pi)(\mathbb{C})$ ) consisting of the fibers of $\pi_{k}(\mathbb{C})\left(\right.$ resp. $\left.\pi_{k}^{R}(\mathbb{C})\right)$ having dimension greater than $\rho_{k}^{1}(g)+3$. Assume it contains an irreducible component of $H_{k}(\pi)(\mathbb{C})$. Since such component dominates $S(\mathbb{C})$ it would follow that it has dimension more than

$$
3 g-3+\rho_{k}^{1}(g)+3=2 g+2 k-2
$$

a contradiction. Hence it follows that $H_{k}^{\prime}(\pi)(\mathbb{C})\left(\right.$ resp. $\left.H_{k}^{\prime R}(\pi)(\mathbb{C})\right)$ does not contain irreducible components of $H_{k}(\pi)(\mathbb{C})\left(\right.$ resp. $\left.H_{k}^{R}(\pi)(\mathbb{C})\right)$, hence its dimension is less than $2 g+2 k-2$. Moreover, those subspaces are invariant under complex conjugation, hence they are defined over $\mathbb{R}$. So we obtain closed subschemes $H_{k}^{\prime}(\pi)$ and 
$H_{k}^{\prime R}(\pi)$ of $H_{k}(\pi)$ defined over $\mathbb{R}$ and we obtain

$$
\operatorname{dim}\left(H_{k}^{\prime}(\pi)(\mathbb{R})\right)<2 g+2 k-2
$$

and

$$
\operatorname{dim}\left(H_{k}^{\prime R}(\pi)(\mathbb{R})\right)<2 g+2 k-2 .
$$

For a complex curve $X_{s}$ corresponding to a general point $s$ on $S(\mathbb{C})$ it follows from Brill-Noether Theory that $W_{k}^{r}(X)$ has dimension

$$
\rho_{k}^{r}(g)=g-(r+1)(g-k+r)
$$

(if $\rho_{k}^{r}(g) \geq 0$, otherwise it is empty). In case $r \geq 2$ this gives rise to a subspace of $\pi_{k}^{-1}(s)$ (resp. $\left.\pi_{k}^{\prime-1}(s)\right)$ of dimension

$$
\rho_{k}^{r}(g)+2(r-1)+3<\rho_{k}^{1}(g)+3 .
$$

Let $H_{k}^{2}(\pi)(\mathbb{C})\left(\right.$ resp. $\left.H_{k}^{2, R}(\pi)(\mathbb{C})\right)$ be the closed subspace of $H_{k}(\pi)(\mathbb{C})\left(\right.$ resp. $\left.H_{k}^{R}(\pi)(\mathbb{C})\right)$ corresponding to linear systems $g_{k}^{1}$ that are not complete. Then we obtain that those are not components of $H_{k}(\pi)(\mathbb{C})$ (resp. $H_{k}^{R}(\pi)(\mathbb{C})$ ) because they have dimension less than $2 g+2 k-2$. Again $H_{k}^{2}(\pi)$ and $H_{k}^{2, R}(\pi)$ are defined over $\mathbb{R}$ and we find

$$
\operatorname{dim}\left(H_{k}^{2}(\pi)(\mathbb{R})\right)<2 g+2 k-2
$$

and

$$
\operatorname{dim}\left(H_{k}^{2, R}(\pi)(\mathbb{R})\right)<2 g+2 k-2 .
$$

Pro of of The ore m 4.4. In case $\rho_{k}^{1}(g)<0$ then from $\operatorname{dim}\left(H_{k}(\pi)(\mathbb{R})\right)<3 g-3$ part 1 follows. Also in case $s=0$ one has $\operatorname{dim}\left(H_{k}^{R}(\pi)(\mathbb{R})\right)<3 g-3$, hence using Lemma 4.3 the first statement of part 3 follows. So assume $\rho_{k}^{1}(g) \geq 0$.

By Theorem 3.2 there is a real curve $X$ of topological type $(g, s, a)$ having a morphism $f: X \rightarrow \mathbb{P}^{1}$ of degree $k$ having topological degree $\underline{\delta}$. Let $\pi: \mathcal{C} \rightarrow S$ be a suited family for $X$ then $f$ defines $[f] \in H_{k}(\pi)(\mathbb{R})$. From the previous consideration it follows we can assume

$$
[f] \notin H_{k}^{\prime}(\pi)(\mathbb{R}) \cup H_{k}^{2}(\pi)(\mathbb{R}) .
$$

From $[f] \notin H_{k}^{\prime}(\pi)(\mathbb{R})$ it follows $\operatorname{dim}\left(\pi_{k}\left(H_{k}(\pi)(\mathbb{R})\right)\right)=3 g-3$ and from $[f] \notin H_{k}^{2}(\pi)(\mathbb{R})$ it follows $f$ corresponds to a complete $g_{k}^{1}$. Hence a general element of $\pi_{k}\left(H_{k}(\pi)(\mathbb{R})\right)$ corresponds to a real curve $X^{\prime}$ of type $(g, s, a)$ with

$$
\operatorname{dim}\left(W_{k}^{1}\left(X^{\prime}\right)(\underline{\delta})\right) \geq \rho_{k}^{1}(g) .
$$

Also $\operatorname{dim}\left(H_{k}(\pi)(\mathbb{R})\right)=2 g+2 k-2$ implies we obtain

$$
\operatorname{dim}\left(W_{k}^{1}\left(X^{\prime}\right)(\underline{\delta})\right)=\rho_{k}^{1}(g)
$$

proving part 2.

In case $s=0$ and $k \equiv g+1(\bmod 2)$, by Theorem 3.4 there exists a real curve $X$ of topological type $(g, 0,1)$ having a real morphism $f: X \rightarrow R_{0}$ of degree $k$. Now we find $[f] \in H_{k}^{R}(\pi)(\mathbb{R})$ and we can assume

$$
[f] \notin H_{k}^{\prime R}(\pi)(\mathbb{R}) \cup H_{k}^{2, R}(\pi)(\mathbb{R}) .
$$

As in the previous case we obtain the second statement of part 3 (again we also use Lemma 4.3). 
Remark 4.6 The proof of Theorem 4.4 is intensively based on known dimension statements in the complex case. In order to be able to use those statements it is very important to know that $\operatorname{dim}\left(H_{k}(\pi)\right)=2 k+2 g-2$ implies $\operatorname{dim}\left(H_{k}(\pi)(\mathbb{R})\right)=2 k+2 g-2$. To make this conclusion it is crucial to know that $H_{k}(\pi)(\mathbb{C})$ is smooth. In his paper [7] mentioned in Remark 3.5 the author uses similar arguments to obtain statements as those in Theorem 4.4 but without considering the topological degree. However the arguments are applied on the moduli space itself. Although the author claims the locus of $k$-gonal curves on the moduli space is smooth this is not clear (and in general it is not true). Moreover for his arguments he needs that the closure of that locus is smooth at the hyperelliptic or trigonal locus. To solve this technical problem one should use a suited family containing a hyperelliptic or trigonal curve and use the space parameterizing linear systems $g_{k}^{1}$ on fibers of that family. Indeed, according to [2] that space is smooth. The construction of that space uses the relative Picard scheme and we did not use that space in our arguments in order to be able to use arguments also applicable in case $s=0$. Also in his paper [5, end of the proof of Theorem 0.1] the author uses similar arguments but again not all details are described completely. In particular the author uses an argument on Hurwitz schemes but it is not clear from it that one obtains real coverings with only simple ramification (in particular, that one obtains a point in the smooth locus of the compactified Hurwitz scheme). Those arguments in [5] can be replaced by using the Hilbert scheme parameterizing morphisms and the details described in this section.

In his paper [7] the author also shows that (with the same remarks on the proof as above), in case $\rho_{k}^{1}(g)<0$, a general $k$-gonal real curve of topological type $(g, s, a)$ has a unique linear system $g_{k}^{1}$. This fact can be obtained from our arguments (and fixing the topological degree of the linear system). As in [7] one uses that a general complex $k$-gonal curve has a unique $g_{k}^{1}$ (see [3]). Since a general complex $k$-gonal curve has no multiple $g_{k}^{1}$ (see [22]) one also obtains a similar result for general real $k$-gonal curves fixing the topological degrees.

We return to Example 4.2, finishing the case of curves of topological type $(4,1,0)$. A non-hyperelliptic complex curve $X$ of genus 4 is trigonal. By Riemann-Roch, in case $g$ is a $g_{3}^{1}$ on $X$ then $\left|K_{X}-g\right|=h$ is also a $g_{3}^{1}$ on $X$ and it is well known that $X$ has no other linear systems $g_{3}^{1}$. Hence $X$ has either two (in case $g \neq h$ ) or one (in case $g=h$ ) linear systems $g_{3}^{1}$.

Proposition 4.7 Let $X$ be a non-hyperelliptic real curve of topological type $(4,1,0)$. Then $X$ has two real linear systems $g_{3}^{1}$. One of them has topological degree (3), the other one has topological degree (1).

Pro of. Let $T$ be a real Teichmüller space of real curves of topological type $(4,1,0)$ and let $\pi_{T}: \mathcal{X} \rightarrow T$ be the associated universal family. This space $T$ is a connected real manifold of dimension 9 and all real curves of topological type $(4,1,0)$ do occur as fibers of that family. Inside $T$ the closed subspace $H$ of points having hyperelliptic fibers for $\pi_{T}$ has codimension 2, hence $T \backslash H$ is still connected. Inside $T \backslash H$ we consider $T(1)$ (resp. $T(3))$ being the subspace of points such that the fiber $X$ for $\pi_{T}$ has a morphism $f: X \rightarrow \mathbb{P}^{1}$ of degree 3 of topological degree (1) (resp. (3)). From [13] we know $T(3)=T \backslash H$ and we need to prove $T(1)=T \backslash H$ too. Because of Theorem 3.2 we know $T(1) \neq \emptyset$, we assume that $T(1) \neq T \backslash H$.

The fiber of a point belonging to the closure of $T(1)$ in $T \backslash H$ is a real curve $X$ that is the limit of real curves $X_{t}$ having a morphism $f_{t}: X_{t} \rightarrow \mathbb{P}^{1}$ of topological degree (1). This corresponds to a linear system $g_{3}^{1}(t)$ on $X_{t}$ and the limit is a linear system $g_{3}^{1}$ on $X$. Since $X$ is not hyperelliptic this linear system has no base points and it is complete, hence it corresponds to a morphism $f: X \rightarrow \mathbb{P}^{1}$ being a limit of those morphisms $f_{t}$, hence $f$ has topological degree (1) too. This implies $T(1)$ is closed in $T \backslash H$ hence it is not open in $T \backslash H$ since $T \backslash H$ is connected. Therefore $T(1)$ has a boundary point $t_{0}$ in $T \backslash H$, let $X_{0}$ be the fiber of $\pi_{T}$ above $t_{0}$. Now let $\pi: \mathcal{C} \rightarrow S$ be a suited morphism of curves for $X_{0}\left(\right.$ let $s_{0} \in S(\mathbb{R})$ such that $\left.\pi^{-1}\left(s_{0}\right)=X_{0}\right)$.

Let $\pi_{3}: H_{3} \rightarrow S$ be the parameterspace for all coverings of degree 3 to $\mathbb{P}^{1}$ of fibers of $\pi$ above points on $S$. Let $H_{3}(1)$ (resp. $H_{3}(3)$ ) be the closed open subsets of coverings of topological degree (1) (resp. (3)). For a covering $f: X \rightarrow \mathbb{P}^{1}$ we write $L_{f}$ to denote the corresponding invertible sheaf. For $[f] \in H_{3}$ it follows from the deformation theory of Horikawa that $d_{[f]}\left(\pi_{3}\right)$ is surjective if and only if $\operatorname{dim}\left(H^{0}\left(L_{f}^{\otimes 2}\right)\right)=3$ (indeed $H^{0}\left(X, N_{f}\right) \rightarrow H^{1}\left(X, T_{X}\right)$ is the tangent map of $\pi_{3}$ at $\left.[f]\right)$. In that case for $x=\pi_{3}([f])$ there is a neighborhood $U$ of $x$ in $S$ such that $U \subset \operatorname{im}\left(\pi_{3}\right)$. In case $\operatorname{dim}\left(H^{0}\left(L_{f}^{\otimes 2}\right)\right)>3$ then $L_{f}^{\otimes 2} \cong \omega_{\pi^{-1}(x)}$, hence $L_{f}$ is half-canonical.

Let $f_{0}: X_{0} \rightarrow \mathbb{P}^{1}$ be the morphism of degree 3 on $X_{0}$ of topological degree (1). For each classical neighborhood $U$ of $s_{0}$ in $S$ there exists $s \in U \cap S(\mathbb{R})$ such that $\pi^{-1}(s)=\pi_{T}^{-1}(t)$ for some $t \notin T(1) \cup H$, hence $U \nsubseteq \pi_{3}\left(H_{3}(1)\right)$. From the previous description of the tangent map of $\pi_{3}$ it follows $L_{f_{0}}$ is half-canonical on $X_{0}$. 
In particular $X_{0}$ has no $g_{3}^{1}$ associated to an invertible sheaf different from $L_{f_{0}}$ and this would imply there is no morphism $f: X_{0} \rightarrow \mathbb{P}^{1}$ of topological degree (3), hence $t_{0} \notin T(3)$ contradicting $T(3)=T \backslash H$.

Remark 4.8 In contrast with our Theorem 4.4 it is already mentioned that for each topological type $(4, s, a) \neq$ $(4,1,0)$ there is a general real curve $X$ having no real $g_{3}^{1}$. In [9] it is also proved that for each topological type $(8, s, a) \neq\{(8,1,0),(8,0,1)\}$ there is a general real curve $X$ having no real $g_{5}^{1}$ (while Theorem 4.4 implies there is a general real curve $X$ having a real $g_{5}^{1}$ ). On the other hand, the main result of [13] implies that there is no general real curve $X$ of topological type $(8,1,0)$ having no base point free $g_{5}^{1}$ of topological degree $(5)$. For real curves without real points it is proved in [20] that for each genus $g$ there exist general real curves $X$ of topological type $(g, 0,1)$ such that $X_{k}^{1}(X)(\mathbb{R}) \backslash W_{k}^{1}(X)(\mathbb{R})^{+}$is empty for each $k \leq g$. Our Theorem 4.4 implies that there exist general real curves of topological type $(g, g-1,0)$ having a base point free $g_{g-1}^{1}$ of topological degree $(1, \ldots, 1)$. From forthcoming work of the first author it follows that there also exist general real curves $X$ of topological type $(g, g-1,0)$ having no such linear system $g_{g-1}^{1}$ and many similar statements.

\section{Real 4-gonal curves having a $g_{4}^{1}$ with no totally non-real divisor and no component of non-zero degree}

Definition 5.1 Let $X$ be a real curve and let $f: X \rightarrow \mathbb{P}^{1}$ be a morphism defined over $\mathbb{R}$. We define the covering number $k(f)$ as follows. Consider the associated map $f(\mathbb{R}): X(\mathbb{R}) \rightarrow \mathbb{P}^{1}(\mathbb{R})$. If $f(\mathbb{R})$ is not surjective then $k(f)=0$. Otherwise $k(f)$ is the minimal number $k$ such that there exist connected components $C_{1}, \ldots, C_{k}$ of $X(\mathbb{R})$ such that $f\left(C_{1} \cup \cdots \cup C_{k}\right)=\mathbb{P}^{1}(\mathbb{R})$.

Of course, in case $X(\mathbb{R})=\emptyset$ then $k(f)=0$ by definition. Also $k(f)=1$ if there is a connected component $C$ of $X(\mathbb{R})$ such that $\delta_{C}(f) \geq 1$. In particular $k(f)=1$ in case $\operatorname{deg}(f)$ is odd. Also in case $\operatorname{deg}(f)=2$ then $k(f)$ is equal to 0 or to 1 . So the case with $\operatorname{deg}(f)=4$ is the first interesting case to study the possible values for $k(f)$. In the next theorem we prove there are no further restrictions on $k(f)$ for gonality 4 .

Theorem 5.2 Let $(g, s, a)$ be an admissible topological type for real curves with $s \geq 1$. Let $1 \leq k \leq s$. There exists a real curve $X$ of topological type $(g, s, a)$ such that there is a covering $f: X \rightarrow \mathbb{P}^{1}$ of degree 4 defined over $\mathbb{R}$ such that $k(f)=k$ and for each connected component $C$ of $X(\mathbb{R})$ one has $\delta_{C}(f)=0$.

Proof. We first prove the case of $M$-curves $(s=g+1$, in particular $a=0)$ with $k=g+1$.

In case $g$ is even take two hyperelliptic $M$-curves $Y_{1}$ and $Y_{2}$ of genus $g^{\prime}=g / 2$ having double coverings $f_{i}: Y_{i} \rightarrow \mathbb{P}^{1}$ defined over $\mathbb{R}$ with the following properties. Let $C_{0}^{i}, \ldots, C_{g^{\prime}}^{i}$ be the connected components of $Y_{i}(\mathbb{R})$ and let $I_{j}^{i}=f_{i}\left(C_{j}^{i}\right)$ then $I_{j_{1}}^{1}$ intersects $I_{j_{2}}^{2}$ if and only if one of the following holds:

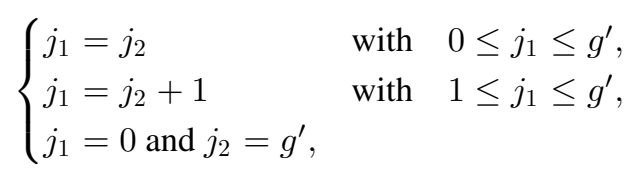

and the non-empty intersections are connected (see Figure 9 with $g^{\prime}=2$ ).

Let $t \in I_{0}^{1} \cap I_{0}^{2}$ and take $p_{i} \in f_{i}^{-1}(t)$. Let $X_{0}=\left(Y_{0} \cup Y_{1}\right)_{p_{1}=p_{2}}$. It is a stable real curve of genus $g$ having a node $p=\left(p_{1}=p_{2}\right)$ and a morphism $f: X_{0} \rightarrow \mathbb{P}^{1}$ of degree 4 defined over $\mathbb{R}$. Locally at $p$ the curve $X_{0}$ is defined over $\mathbb{R}$ by the equation $x^{2}-y^{2}=0$ and the morphism is given by $(x, y) \mapsto x$. Using a local deformation over $\mathbb{R}$ given by $x^{2}-y^{2}=t$ and gluing with other local coordinates one obtains a curve $X_{t}$ defined over $\mathbb{R}$ having a covering $f_{t}: X_{t} \rightarrow \mathbb{P}^{1}$ of degree 4 defined over $\mathbb{R}$ such that $g\left(X_{t}\right)=2 g^{\prime}=g$. (The details are as in Construction I.) The components of $X_{t}(\mathbb{R})$ are deformations $C_{j}^{i}(t)$ of $C_{j}^{i}$ for $1 \leq j \leq g^{\prime}$ and $i=1 ; 2$ and of $\left(C_{0}^{1} \cup C_{0}^{2}\right)_{p_{1}=p_{2}}$ which is one component $C_{0}(t)$. Clearly $f_{t}\left(C_{0}(t)\right)$ is a deformation of $I_{0}^{1} \cup I_{0}^{2}$ and $f_{t}\left(C_{j}^{i}(t)\right)$ is a deformation of $I_{j}^{i}$ for $i=1 ; 2,1 \leq j \leq g^{\prime}$. It follows that their union is equal to $\mathbb{P}^{1}(\mathbb{R})$ but omitting one of them does not cover $\mathbb{P}^{1}(\mathbb{R})$. It follows $s\left(X_{t}\right)=2 g^{\prime}+1=g+1$ (hence $X_{t}$ is an $M$-curve) and $k=g+1$.

In case $g$ is odd take two hyperelliptic $M$-curves $Y_{1}$ of genus $g^{\prime}=(g-1) / 2$ and $Y_{2}$ of genus $g^{\prime}+1=(g+1) / 2$. Use the notation $C_{j}^{i}$ for the components of $Y_{i}(\mathbb{R})$ with $0 \leq j \leq g^{\prime}$ in case $i=1$ and $0 \leq j \leq g^{\prime}+1$ in case $i=2$. Let $I_{j}^{i}=f_{i}\left(C_{j}^{i}\right)$ and assume $I_{j_{1}}^{1}$ intersects $I_{j_{2}}^{2}$ for $0 \leq j_{1} ; j_{2} \leq g^{\prime}$ as in the previous case and $I_{g^{\prime}+1}^{2} \subset I_{0}^{1}$ (see Figure 10 with $g^{\prime}=2$ ). 


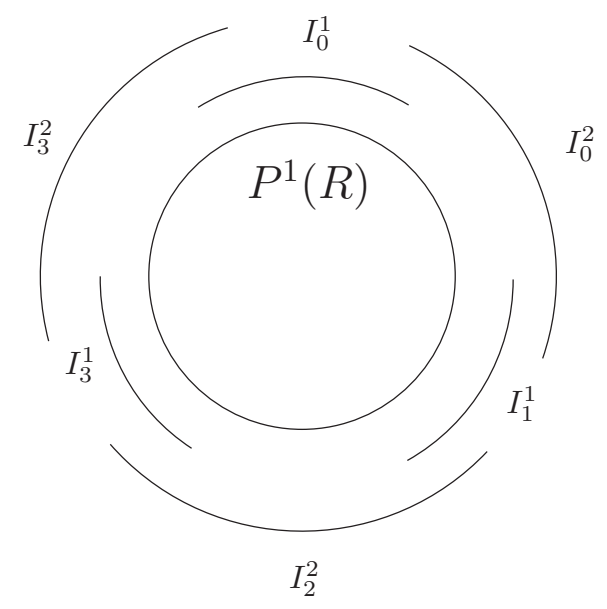

Fig. $9 s=k=g+1$ and $g$ even.

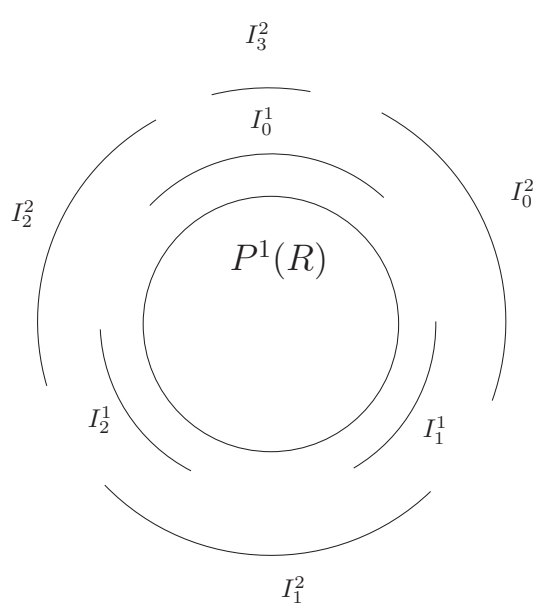

Fig. $10 s=k=g+1$ and $g$ odd.

Let $t \in I_{g^{\prime}+1}^{2}$ and take $p_{i} \in f_{i}^{-1}(t)$. Let $X_{0}$ be as before then arguing as before one obtains an $M$-curve $X_{t}$ of genus $g$ with a covering $f_{t}: X_{t} \rightarrow \mathbb{P}^{1}$ of degree 4 defined over $\mathbb{R}$ such that the components of $X_{t}(\mathbb{R})$ are deformations $C_{j}^{i}(t)$ for $0 \leq j \leq g^{\prime}$ and $i=1 ; 2$ (with $j \neq 0$ for $\left.i=1\right)$ and $C(t)$ of $\left(C_{0}^{1} \cup C_{g^{\prime}+1}^{2}\right)_{p_{1}=p_{2}}$. The images $f_{t}\left(C_{j}^{i}(t)\right)$ are deformations of $I_{j}^{i}$ and the image $f_{t}(C(t))$ is a deformation of $I_{0}^{1}$. It follows $k=g+1$.

Now we are going to prove the theorem for $M$-curves in case $k<g+1$. From the previous part of the proof we obtain the existence of an $M$-curve $Y$ of genus $k-1$ having a covering $f_{Y}: Y \rightarrow \mathbb{P}^{1}$ of degree 4 defined over $\mathbb{R}$ satisfying the following property. Let $C_{1}, \ldots, C_{k}$ be the components of $Y(\mathbb{R})$, let $I_{i}=f_{Y}\left(C_{i}\right)$ then $I_{i}$ intersects $I_{j}$ for $i \neq j$ if and only if

$$
\begin{cases}j=i+1 & \text { for } \quad 1 \leq i \leq k-1 \\ j=k & \text { and } \quad i=1\end{cases}
$$

and the non-empty intersections are connected. In case $k=1$ then $Y(\mathbb{R})$ has a unique component $C_{1}$ dominating $\mathbb{P}^{1}(\mathbb{R})$ such that $\delta_{f_{Y}}\left(C_{1}\right)=0$ and there is a connected closed subset $I \subset \mathbb{P}^{1}(\mathbb{R})$ such that $x \in I$ if and only if $f_{Y}^{-1}(x) \subset Y(\mathbb{R})$.

Let $c_{1}, \ldots, c_{g-k+1}$ be different points on $I_{1} \cap I_{2}$ in this order (with $c_{1}$ most close to $I_{1} \backslash I_{2}$ ) and let $f_{Y}^{-1}\left(x_{i}\right) \cap$ $C_{1}=\left\{p_{i 1}, p_{i 2}\right\}$ (see Figure 11 with $k=4$ and $g=6$ ). In case $k=1$ those are points in the inner part of $I$. Let $X_{0}=Y_{p_{i 1}=p_{i 2} \text { for } 1 \leq i \leq g-k+1}$. Then $X_{0}$ is defined over $\mathbb{R}$ and it has a covering $f_{0}: X_{0} \rightarrow \mathbb{P}^{1}$ defined over $\mathbb{R}$ of degree 4 . Locally at the node $p_{i}=\left(p_{i 1}+p_{i 2}\right)$ the curve $X_{0}$ is defined over $\mathbb{R}$ by $x^{2}-y^{2}=0$ and the morphism 


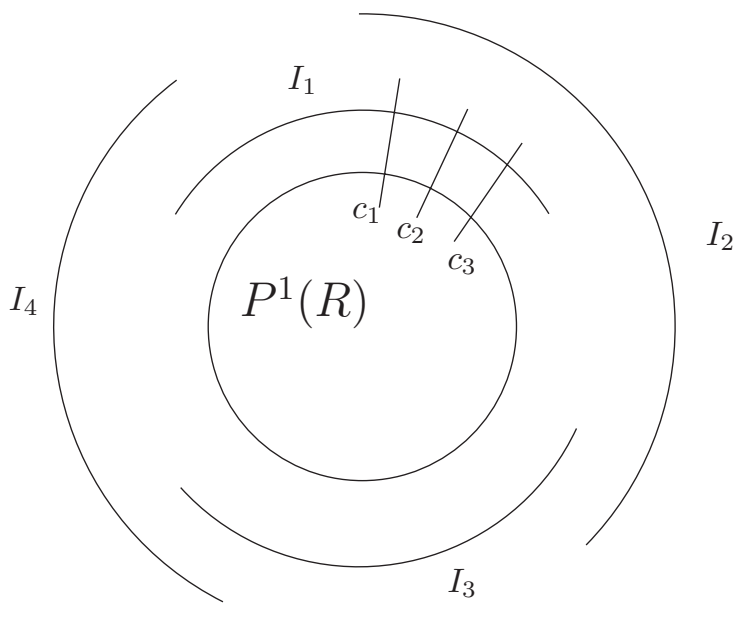

Fig. $11 k<s=g+1$.

by $(x, y) \mapsto x$. Using local deformations over $\mathbb{R}$ by the equation $x^{2}-y^{2}=t$ with $t \geq 0$ one obtains two new real ramification points close to $c_{i}$. Gluing one obtains a curve $X_{t}$ defined over $\mathbb{R}$ having a morphism $f_{t}: X_{t} \rightarrow \mathbb{P}^{1}$ of degree 4 defined over $\mathbb{R}$ with $g\left(X_{t}\right)=g$. ( This is similar to applying Construction I with real ramification $g-k+1$ times.) For each $2 \leq i \leq k$ there is a component $C_{i}(t)$ of $X_{t}(\mathbb{R})$ that is a deformation of $C_{i}$. Because of the chosen local deformations, the deformation of $C_{1}$ is a union of $g-k+2$ components $C_{1}(t), C_{1}^{\prime}(t), \ldots, C_{g-k+1}^{\prime}(t)$. It follows $s\left(X_{t}\right)=g+1$ hence $X_{t}$ is an $M$-curve. The images $f_{t}\left(C_{i}(t)\right)$ are deformations of $I_{i}$ for $2 \leq i \leq t$, the image $f_{t}\left(C_{1}(t)\right)$ is a deformation of the connected component of $I_{1} \backslash\left\{c_{1}\right\}$ containing $I_{1} \backslash I_{2}$, the images of $f_{t}\left(C_{i}^{\prime}(t)\right)$ are deformations of the interval between $c_{i}$ and $c_{i+1}$ on $I_{1}$ for $1 \leq i \leq g-k$ and a deformation of the connected component of $I_{1} \backslash\left\{c_{g-k+1}\right\}$ not containing $I_{1} \backslash I_{2}$ for $i=g-k+1$.

If follows $C_{1}(t), \ldots, C_{k}(t)$ is the only subset of $k$ components $C$ of $X_{t}(\mathbb{R})$ such that the union of the intervals $f_{t}(C)$ equals $\mathbb{P}^{1}(\mathbb{R})$. This implies $k\left(f_{t}\right)=k$.

Now we finish the proof for the orientable case $a=0$ in case $X$ is not an $M$-curve (in particular $s \leq g-1$ ). Let $b=(g+1-s) / 2$, which is an integer. From the prevous part of the proof we obtain the existence of an $M$-curve $Y$ of genus $g-b=s+b-1$ (hence $Y(\mathbb{R})$ has $s+b$ connected components) having a covering $f_{Y}: Y \rightarrow \mathbb{P}^{1}$ of degree 4 defined over $\mathbb{R}$ satisfying the following property. Let $C_{1}, \ldots, C_{s+b}$ be the components of $Y(\mathbb{R})$ and let $I_{i}=f_{Y}\left(C_{i}\right)$. Then $I_{j}$ and $I_{j^{\prime}}$ with $1 \leq j^{\prime}<j \leq k+b$ do have a point in common if and only if

$$
\left\{\begin{array}{l}
j^{\prime}=j+1 \quad \text { for } \quad 1 \leq j \leq k+b-1, \\
j=k+b \quad \text { and } \quad j^{\prime}=1,
\end{array}\right.
$$

and the non-empty intersections are connected and $I_{k+b+1}, \ldots, I_{s+b}$ is contained in $I_{1} \backslash\left(I_{2} \cup I_{k+b}\right)$ (see Figure 12 with $k+b=4$ and $s+b=6$ ).

For $1 \leq j \leq b$ let $p_{j} \in C_{j}$ and $q_{j} \in C_{j+1}$ with $f_{Y}\left(p_{j}\right)=f_{Y}\left(q_{j}\right) \in I_{j} \cap I_{j+1}$. Let $X_{0}=Y_{p_{j}=q_{j} \text { for } 1 \leq j \leq b \text {. }}$ Using locally real deformations at the nodes $p_{j}=q_{j}$ one obtains a real curve $X_{t}$ of genus $g(Y)+b=g$ and a covering $f_{t}=X_{t} \rightarrow \mathbb{P}^{1}$ of degree 4 defined over $\mathbb{R}$. The components of $X_{t}(\mathbb{R})$ are a deformation $C(t)$ of

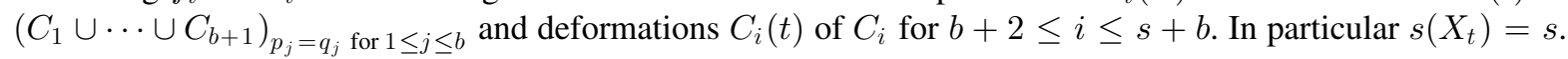
The image $f(C(t))$ is a deformation of $I_{1} \cup \cdots \cup I_{b+1}$ and the image $f\left(C_{i}(t)\right)$ for $b+2 \leq i \leq s+b$ are deformations of $I_{i}$. It follows that $k\left(f_{t}\right)=k$.

Finally we consider the non-orientable case (hence $a=1)$. First assume $s \equiv g(\bmod 2)$. Let $Y$ be an orientable curve of genus $g-1$ with $s(Y)=s$ such that $Y$ has a covering $f_{Y}: Y \rightarrow \mathbb{P}^{1}$ of degree 4 defined over $\mathbb{R}$ with $k\left(f_{Y}\right)=k$. Let $c \in \mathbb{P}^{1}(\mathbb{R})$ such that $f_{Y}^{-1}(c)$ is not a totally real divisor and let $P+\bar{P} \subset f_{Y}^{-1}(c)$ for a non-real pair $P+\bar{P}$. Let $X_{0}=Y_{P=\bar{P}}$, it is a real curve of genus $g+1$ having a covering of degree 4 defined over $\mathbb{R}$. Locally at the node $P=\bar{P}$ the curve is defined over $\mathbb{R}$ by $x^{2}+y^{2}=0$ and the morphism by $(x, y) \mapsto x$. Take a local deformation of the the type $Z\left(x^{2}+y^{2}-t\right)$ with $t<0$ and glue it to obtain a real curve $X_{t}$ (this corresponds to Construction II without real ramification). Clearly $X_{t}$ is non-orientable, $g\left(X_{t}\right)=g, s\left(X_{t}\right)=s$ and it has a covering $f_{t}: X_{t} \rightarrow \mathbb{P}^{1}$ defined over $\mathbb{R}$ of degree 4 with $k\left(f_{Y}\right)=k$. Finally assume $s \equiv g+1$ 


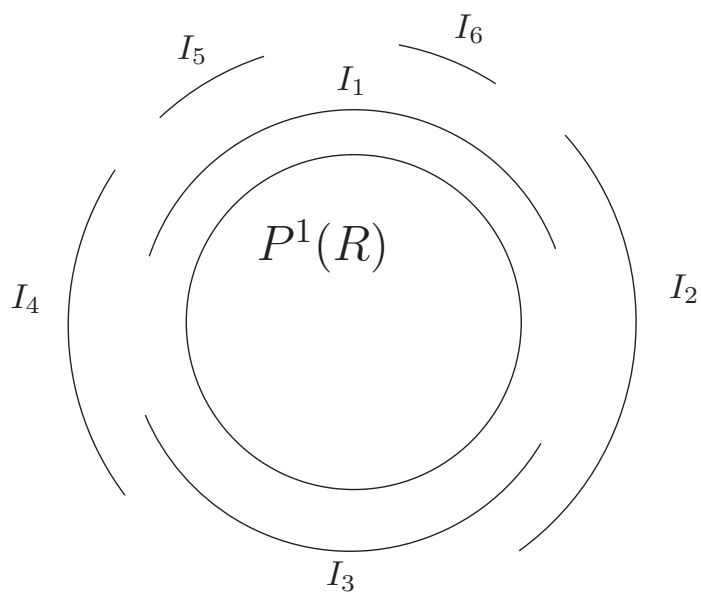

Fig. $12 a=0 ; s<g+1$.

(mod 2). Since $s \leq g-1$ we can take a non-orientable real curve $Y$ of genus $g-1$ such that $s(Y)=s$ and there is a covering $f_{Y}: Y \rightarrow \mathbb{P}^{1}$ of degree 4 defined over $\mathbb{R}$ with $k\left(f_{Y}\right)=k$. Repeating the previous construction one obtains the curve $X_{t}$ one is looking for.

\section{References}

[1] N. L. Alling and N. Greenleaf, Foundations of the Theory of Klein Surfaces Vol. 219 (Lecture Notes in Mathematics, Springer, 1971).

[2] E. Arbarello and M. Cornalba, Su una congettura di Petri, Comment. Math. Helv. 66, 1-38 (1981).

[3] E. Arbarello and M. Cornalba, Footnotes to a paper of Beniamino Segre, Math. Ann. 256, 341-362 (1981).

[4] E. Arbarello, M. Cornalba, P. Griffiths, and J.Harris, Geometry of Algebraic Curves Vol. I (Grundlehren den Math. Wiss. Band 267, Springer, 1985).

[5] E. Ballico, Real algebraic curves and real spanned bundles, Ric. Mat. 50, 223-241 (2001).

[6] E. Ballico, Real curves with fixed gonality and empty real locus, Le Matematiche 60, 129-131 (2005).

[7] E. Ballico, Real ramification points and real Weierstrass points of real projective curves, Glas. Mat. 41, 233-238 (2006).

[8] E. Biswas, On line bundles over real algebraic curves, Bull. Sci. Math. 134, 337-449 (2010).

[9] S. Chaudhary, The Brill-Noether Theorem for Real Algebraic Curves, PhD. thesis, Duke University (1995).

[10] A. F. Costa and M. Izquierdo, On real trigonal Riemann surfaces, Math. Scand. 98, 53-68 (2006).

[11] M. Coppens, Totally non-real divisors in linear systems on smooth real curves, Adv. Geom. 8, 551-555 (2008).

[12] M. Coppens and G. Martens, Linear pencils on real algebraic curves, J. Pure Appl. Algebra 214, 841-849 (2010).

[13] A. Gabard, Sur la representation conforme des surfaces de Riemann à bord et une caractérisation des courbes séparantes, Comment. Math. Helv. 81, 945-964 (2006).

[14] B. H. Gross and J. Harris, Real algebraic curves, Ann. Sci. Éc. Norm. Supér. 14, 157-182 (1981).

[15] A. Grothendieck, Techniques de construction et théorèmes d'existence en géometrie algébrique IV Les schémas de Hilbert, Séminaire Bourbaki 221 (1960/61).

[16] A. Grothendieck, Techniques de construction et théorèmes d'existence en géometrie algébrique V Les schémas de Picard, Séminaire Bourbaki 232 (1961/62).

[17] E. Horikawa, On deformations of holomorphic maps I, J. Math. Soc. Japan 25, 372-396 (1973).

[18] J. Huisman, Real quotient singularities and nonsingular real algebraic curves in the boundary of the moduli space, Compos. Math. 118, 42-60 (1999).

[19] J. Huisman, Real Teichmüller spaces and moduli of real algebraic curves, Contemp. Math. 253, 145-179 (2000).

[20] G. Martens, Minimale Blätterzahl bei Überlagerungen Kleinschen Flächen der projektiven Ebene, Achiv der Math. 30, 481-486 (1978).

[21] J. -Ph.Monnier, Very special divisors on real algebraic curves, Bull. Lond. Math. Soc. (to appear).

[22] B. Segre, Sui moduli delle curve poligonali e sopra un complemento al teorema di esistenza di Riemann, Math. Ann. 100, 537-551 (1928).

[23] E. Sernesi, Deformations of Algebraic Schemes (Grundlehren der Math. Wiss. Band 333, Springer, 2006).

[24] M. Teixidor i Bigas, Half-canonical series on algebraic curves, Transactions of the AMS 302, 99-115 (1987).

[25] G. Weichold, Uber symmetrische Riemann'sche Flächen und die Periodicitätsmoduln zugehörigen Abel'schen Normalintegrale erster Gattung, Zeit. f. Math. u. Phys. 28, 321-351 (1883). 